\title{
Necking, Beading, and Bulging in Soft Elastic Cylinders
}

\author{
Yibin $\mathrm{Fu}^{\mathrm{a}, *}$, Lishuai Jin ${ }^{\mathrm{c}, \mathrm{b}}$, Alain Goriely ${ }^{\mathrm{d}}$ \\ ${ }^{a}$ School of Computing and Mathematics, Keele University, Staffs ST5 5BG, UK \\ ${ }^{b}$ Department of Mechanics, Tianjin University, Tianjin 300072, China \\ ${ }^{c}$ John A. Paulson School of Engineering and Applied Sciences, Harvard University, Cambridge, MA 02138, USA \\ ${ }^{d}$ Mathematical Institute, University of Oxford, Oxford, OX2 6GG, UK
}

\begin{abstract}
Due to surface tension, a beading instability takes place in a long enough fluid column that results in the breakup of the column and the formation of smaller packets with the same overall volume but a smaller surface area. Similarly, a soft elastic cylinder under axial stretching can develop an instability if the surface tension is large enough. This instability occurs when the axial force reaches a maximum with fixed surface tension or the surface tension reaches a maximum with fixed axial force. However, unlike the situation in fluids where the instability develops with a finite wavelength, for a hyperelastic solid cylinder that is subjected to the combined action of surface tension and axial stretching, a linear bifurcation analysis predicts that the critical wavelength is infinite. We show, both theoretically and numerically, that a localized solution can bifurcate subcritically from the uniform solution, but the character of the resulting bifurcation depends on the loading path. For fixed axial stretch and variable surface tension, the localized solution corresponds to a bulge or a depression, beading or necking, depending on whether the axial stretch is greater than a certain threshold value that is dependent on the material model and is equal to $\sqrt[3]{2}$ when the material is neo-Hookean. At this single threshold value, localized solutions cease to exist and the bifurcation becomes exceptionally supercritical. For either fixed surface tension and variable axial force, or fixed axial force and variable surface tension, the localized solution corresponds to a depression or a bulge, respectively. We explain why the bifurcation diagrams in previous numerical and experimental studies look as if the bifurcation were supercritical although it was not meant to. Our analysis shows that beading in fluids and solids are fundamentally different. Fluid beading resulting from the Plateau-Rayleigh instability follows a supercritical linear instability whereas solid beading in general is a subcritical localized instability akin to phase transition.
\end{abstract}

Keywords: Beading instability, Localization, Bifurcation, Nonlinear elasticity.

\section{Introduction}

Surface tension operates in fluids and is the driving force behind many fascinating phenomena from the formation of droplets to the motion insects, such as water striders, atop a liquid (De Gennes et al., 2004; Bush \& Hu, 2006). It is of fundamental importance for many biological and physical phenomena and is one of the most studied areas of fluid mechanics (Levich \& Krylov, 1969). A well-known phenomenon associated with surface tension is the Plateau-Rayleigh instability where a cylindrical column of fluids destabilizes to form droplets, hence lowering its overall energy by reducing its surface area (Plateau, 1873; Rayleigh, 1892).

\footnotetext{
${ }^{*}$ Corresponding author

Email address: y.fu@keele.ac.uk (Yibin Fu)
} 

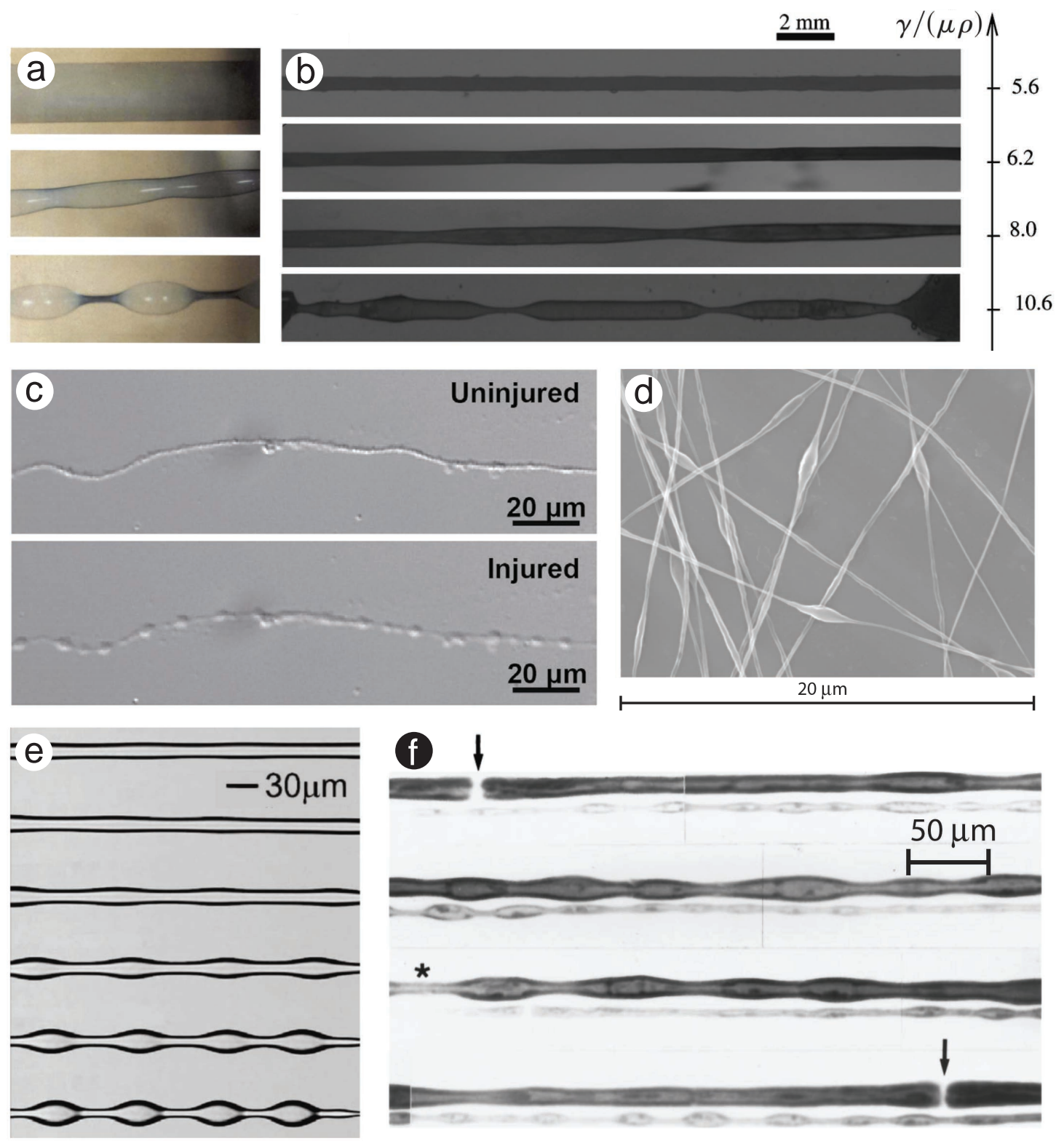

Figure 1: Example of elastocapillary beading, bulging, pearling, and peristaltic instability in soft solids. (a) Beading in a shrinking cylindrical acrylamide gel immersed in an acetone-water mixture (Matsuo \& Tanaka, 1992). (b) Beading instability of a soft agar cylindrical gels immersed in toluene (Mora et al., 2010). (c) Axonal swelling due to mechanical trauma (Hemphill et al., 2015). (d) Beaded nanofibers formed during electrospinning (Fong et al., 1999). (e) Beading due to thinning in polymer nanofibers (Sattler et al., 2008). (f) Beading in a single myelinated fiber teased from a rat sciatic nerve stretched with a weight of $4.5 \mathrm{~g}$ (Markin et al., 1999).

In solids, the situation is quite different. For most materials, surface tension is negligible compared with typical elastic moduli, in which case the contribution of surface tension to the statics and dynamics can be safely ignored. Indeed, surface tension operates in solids at an elastocapillary length scale given by $\gamma / \mu$ where $\gamma$ is the surface tension and $\mu$ is the shear modulus (Style et al., 2017; Bico et al., 2018; Mozaffari et al., 2019). This length scale for many solid is below the atomic level and does not enter the continuum theory in any meaningful way. However, in the last decade, there has been an increasing interest in extremely soft solids such as polymer gels and biological 
tissues. For such materials the elastocapillary length can be microns or even millimeters and it becomes comparable to other typical lengths in the systems. As shown in Fig. 1, it has been observed that in shrinking gels (Matsuo \& Tanaka, 1992), dilute polymer solutions (Sattler et al., 2008), nerve fibers (Bar-Ziv \& Moses, 1994; Nelson \& Powers, 1995; Ochs et al., 1997; Bar-Ziv et al., 1997, 1999; Markin et al., 1999; Pullarkat et al., 2006; Al-Izzi et al., 2018), and polymer fibers produced by electrospinning (Fong et al., 1999; Naraghi et al., 2007) surface tension plays a major role in the so-called beading/pearling instability in which localized axisymmetric beads form on the cylindrical fibers.

Understanding the mechanisms leading to such a shape transformation is of major importance since, for instance, axonal beading is triggered by mechanical trauma (Kilinc et al., 2008, 2009; Hemphill et al., 2015; Goriely et al., 2015; Lang et al., 2017), and is also believed to precede axonal atrophy in Alzheimer's disease, Parkinson's disease, and other neurodegenerative conditions (Datar et al., 2019).

The instability of a pulled elastic cylinder subject to surface tension has been examined using linear elasticity theory by Barriere et al. (1996), Boudaoud \& Chaïeb (2003) and Mora et al. (2010), and more recently using nonlinear elasticity theory by Ciarletta \& Ben Amar (2012), Taffetani \& Ciarletta (2015), Xuan \& Biggins (2016). All these studies conclude that the critical bifurcation mode has infinite wavelength (or zero wave number) in the axial direction. The central question is to study this instability. Since a new solution cannot emerge with such a wavelength, what solution, if any, emerges at the bifurcation? The analogy with the Plateau-Rayleigh instability has led many authors to look for periodic solutions after the bifurcation. However, the general theory of dynamical systems (Haragus \& Iooss, 2011) suggests that a localized mode, rather than a periodic solution, may bifurcate from the uniform configuration. This contradiction does not seem to have attracted the attention of researchers in the early studies of this particular area of research. For instance, although a weakly nonlinear post-bifurcation analysis was conducted by Taffetani \& Ciarletta (2015), their analysis did not yield the quadratic normal form expected for such localized bifurcation problems. To further confuse the situation, numerical studies based on Abaqus by Henann \& Bertoldi (2014), and the experimental results given by Mora et al. (2010), seem to suggest that the instability is a supercritical bifurcation. A later study by Xuan \& Biggins (2017) did note, for the first time, the analogy between the current problem and other phase-transition-like problems, but the study was focussed on the characterisation of the final "two-phase" state.

The objective of our study is two-fold. Firstly, we conduct an appropriate weakly nonlinear analysis and demonstrate that a localized mode does indeed bifurcate from the uniform state. This theoretical conclusion is further validated through numerical simulations. We will also clarify how the bifurcated solutions would connect to the final "two-phase" solutions observed in previous numerical and experimental studies, and explain why the bifurcation diagrams in these studies look as if the bifurcation were supercritical. Secondly, we wish to highlight the similarities between the beading instability and localized bulging in inflated hyperelastic tubes. The latter problem is now well understood both analytically and experimentally (Wang et al., 2019), and it can help us understand the beading problem for which experiments are harder to conduct.

The rest of this paper is divided into six sections as follows. After formulating the problem in the next Section, we derive in Section 3 the bifurcation condition for a general material model and provide a simple interpretation for the bifurcation condition. This extends what has been achieved in previous studies which focused on the neo-Hookean material model. A weakly nonlinear analysis for three loading scenarios is then carried out in Section 4 and localized solutions are derived explicitly. In Section 5 we compare our theoretical predictions with numerical simulation results obtained using Abaqus, and make observations on fully nonlinear post-bifurcation behaviour based on the analogy with the bulging problem of inflated tubes. After discussing in Section 6 connections 
of our current work with some studies that have only recently appeared, we conclude the paper in Section 7 with a summary.

\section{Formulation}

We consider a hyperelastic solid cylinder that is defined in terms of cylindrical polar coordinates by $R \leq B, 0 \leq \Theta \leq 2 \pi$ and $|Z|<L$, where $B$ is the undeformed radius and $L$ is the half-length. We consider axisymmetric deformations given by

$$
r=r(R, Z), \quad \theta=\Theta, \quad z=z(R, Z),
$$

where $(r, \theta, z)$, are cylindrical polar coordinates in the deformed configuration with $0 \leq \theta \leq 2 \pi$, $r \leq b(z)$ and $|z|<\ell$. The deformed external radius of the cylinder is $b(z)$ and its half-length denoted by $\ell$. The position vectors of a representative material particle are thus given by

$$
\boldsymbol{x}=r \boldsymbol{e}_{r}+z \boldsymbol{e}_{z}, \quad \boldsymbol{X}=R \boldsymbol{E}_{R}+Z \boldsymbol{E}_{Z},
$$

in the undeformed and deformed configurations, respectively, where $\left(\boldsymbol{e}_{r}, \boldsymbol{e}_{\theta}, \boldsymbol{e}_{z}\right)$ and $\left(\boldsymbol{E}_{R}, \boldsymbol{E}_{\Theta}, \boldsymbol{E}_{Z}\right)$ are the usual orthonormal bases attached to cylindrical coordinates. The deformation gradient is given by (Goriely, 2017, p.268)

$$
\mathbf{F}=\frac{\partial r}{\partial R} \boldsymbol{e}_{r} \otimes \boldsymbol{E}_{R}+\frac{\partial r}{\partial Z} \boldsymbol{e}_{r} \otimes \boldsymbol{E}_{Z}+\frac{r}{R} \boldsymbol{e}_{\theta} \otimes \boldsymbol{E}_{\Theta}+\frac{\partial z}{\partial R} \boldsymbol{e}_{z} \otimes \boldsymbol{E}_{R}+\frac{\partial z}{\partial Z} \boldsymbol{e}_{z} \otimes \boldsymbol{E}_{Z} .
$$

The cylinder is assumed to be incompressible with its constitutive behavior described by a generalized neo-Hookean strain-energy function of the form

$$
W=w\left(\hat{I}_{1}\right)
$$

where $\hat{I}_{1}$ is the trace of the left Cauchy-Green strain tensor $\mathbf{F F}^{T}$ (exceptionally, we do not use the traditional notation $I_{1}$ which is reserved for the modified Bessel's function of the first kind to be used later). This class of strain-energy functions has been shown to be suitable for many different materials in tension (Wineman, 2005) and include the neo-Hookean model with $W=\mu / 2\left(\hat{I}_{1}-3\right)$ and the Gent model

$$
W=-\frac{\mu}{2} J_{m} \ln \left(1-\frac{\hat{I}_{1}-3}{J_{m}}\right)
$$

where $\mu$ is the ground state shear modulus and $J_{m}$ is a material constant characterizing material extensibility. The neo-Hookean model is recovered in the limit $J_{m} \rightarrow \infty$. Our analysis can be easily extended to strain-energy functions that also depend on the second principal invariant of $\mathbf{F F}^{T}$, but this is not carried out explicitly in the current paper for the sake of brevity.

The total energy for static axisymmetric solutions is the sum of the elastic energy and the surface energy

$$
\mathcal{E}=\int_{-L}^{L} \int_{0}^{B} 2 \pi R w\left(\hat{I}_{1}\right) d R d Z+2 \pi \gamma \int_{-\ell}^{\ell} b(z) \sqrt{1+\left(b^{\prime}(z)\right)^{2}} d z
$$

where $\gamma$ is the surface tension, a material parameter.

Within the set of axisymmetric solutions, our goal is to determine possible bifurcations from a homogeneous cylindrical solution created by the combined action of an axial force and surface 
tension to a non-homogenous solution. The solid cylinder admits, for all strain-energy functions, a homogeneous deformation (Goriely, 2017, p. 308)

$$
r=\frac{1}{\sqrt{\lambda}} R, \quad \theta=\Theta, \quad z=\lambda Z
$$

with deformation gradient given by

$$
\mathbf{F}=\frac{1}{\sqrt{\lambda}} \boldsymbol{e}_{r} \otimes \boldsymbol{E}_{R}+\frac{1}{\sqrt{\lambda}} \boldsymbol{e}_{\theta} \otimes \boldsymbol{E}_{\Theta}+\lambda \boldsymbol{e}_{z} \otimes \boldsymbol{E}_{Z}
$$

where $\lambda=\ell / L$ is the constant stretch in the $z$-direction.

\subsection{Stream-function formulation}

For the general case when $\mathbf{F}$ is given by (2.3), the problem can be formulated elegantly by introducing a stream function in mixed-coordinates $\phi=\phi(R, z)$ so that

$$
Z=\left.\frac{1}{R} \frac{\partial \phi}{\partial R}\right|_{z}=\frac{1}{R} \phi_{, R}, \quad r^{2}=\left.2 \frac{\partial \phi}{\partial z}\right|_{R}=2 \phi_{, z},
$$

and $\phi_{, R}=\partial \phi / \partial R$ etc; see Carroll (2004), Ciarletta (2011), and Ciarletta \& Ben Amar (2012). The advantage of this formulation is that the incompressibility condition $\operatorname{det} \mathbf{F}=1$ is satisfied exactly for

all deformations, and the problem can be formulated in terms of a single function $\phi$. For instance, it can be readily checked that the homogenous deformation (2.7) corresponds to

$$
\phi=\frac{z R^{2}}{2 \lambda}
$$

In terms of this new function, the deformation gradient (2.3) becomes

$$
\begin{aligned}
& \mathbf{F}=\left(\frac{\phi_{, R z}}{\sqrt{2 \phi_{, z}}}+\frac{\phi_{, z z}}{\phi_{, R z} \sqrt{2 \phi_{, z}}}\left(\frac{\phi_{, R}}{R}-\phi_{, R R}\right)\right) \boldsymbol{e}_{r} \otimes \boldsymbol{E}_{R}+\frac{R \phi_{, z z}}{\phi_{, R z} \sqrt{2 \phi_{, z}}} \boldsymbol{e}_{r} \otimes \boldsymbol{E}_{Z}+\frac{\sqrt{2 \phi_{, z}}}{R} \boldsymbol{e}_{\theta} \otimes \boldsymbol{E}_{\Theta} \\
& +\frac{1}{\phi_{, R z}}\left(\frac{\phi_{, R}}{R}-\phi_{, R R}\right) \boldsymbol{e}_{z} \otimes \boldsymbol{E}_{R}+\frac{R}{\phi_{, R z}} \boldsymbol{e}_{z} \otimes \boldsymbol{E}_{Z} .
\end{aligned}
$$

We can now reformulate the energy in terms of the stream function as

$$
\mathcal{E}\left[\phi_{, R R}, \phi_{, R z}, \phi_{, z z}, \phi_{, R}, \phi_{, z}\right]=2 \pi \int_{-\ell}^{\ell} \int_{0}^{B} \mathcal{U} d R d z+2 \pi \int_{-\ell}^{\ell} \mathcal{V} d z
$$

where the strain energy density $\mathcal{U}$ and surface energy density $\mathcal{V}$ are given by

$$
\mathcal{U}=w\left(\hat{I}_{1}\right) \phi_{, R z}, \quad \mathcal{V}=\gamma \sqrt{\left.2 \frac{\partial \phi}{\partial z}\right|_{B}+\left(\left.\frac{\partial^{2} \phi}{\partial z^{2}}\right|_{B}\right)^{2}} .
$$

The total energy $\mathcal{E}$ is seen to be a functional in its arguments $\phi_{, R R}, \phi_{, R z}, \phi_{, z z}, \phi_{, R}, \phi_{, z}$. It then follows that the Euler-Lagrangian equation is given by (Ciarletta \& Ben Amar, 2012)

$$
\left(\frac{\partial \mathcal{U}}{\partial \phi_{, i j}}\right)_{, i j}-\left(\frac{\partial \mathcal{U}}{\partial \phi_{, k}}\right)_{, k}=0
$$


where $k=R$ or $z, i j=R R, R z$ or $z z$ and we assume the summation convention throughout this paper. The surface energy density $\mathcal{V}$ does not appear in these equations as it is part of the boundary condition at $R=B$ given by

$$
\begin{aligned}
& \frac{\partial \mathcal{U}}{\partial \phi_{, R}}-\left(\frac{\partial \mathcal{U}}{\partial \phi_{, R R}}\right)_{, R}-\left(\frac{\partial \mathcal{U}}{\partial \phi_{, R z}}\right)_{, z}+\left(\frac{\partial \mathcal{V}}{\partial \phi_{, z z}}\right)_{, z z}-\left(\frac{\partial \mathcal{V}}{\partial \phi_{, z}}\right)_{, z}=0 \\
& \frac{\partial \mathcal{U}}{\partial \phi_{, R R}}=0
\end{aligned}
$$

where all expressions are evaluated at $R=B$. We observe that the boundary condition (2.15) corresponds to zero shear stress, whereas (2.14) is equivalent to the condition that the outer surface is subject to a normal traction given by $\gamma$ times the trace of the curvature tensor (Taffetani \& Ciarletta, 2015). In the rest of the paper, we scale all lengths by $B$, stress and pressure by $\mu$, and $\gamma$ by $\mu B$. We use the same letters to denote the scaled quantities. Most of the derivations are carried out with the aid of Mathematica (Wolfram, 2019).

\section{Linear bifurcation analysis}

We look for a solution of the form

$$
\phi=\frac{z R^{2}}{2 \lambda}+R f\left(\frac{R}{\sqrt{\lambda}}\right) \mathrm{e}^{\mathrm{i} k z}
$$

where $k$ is the wave number and the function $f(r)$ is to be determined. On substituting this expression into (2.13)-(2.15) and linearizing in terms of $f$, we obtain

$$
\begin{aligned}
& \triangle_{q_{1}} \triangle_{q_{2}} f(r)=0, \quad 0<r<b, \\
& r^{3} f^{(3)}(r)+2 r^{2} f^{\prime \prime}(r)-r l_{1}(r) f^{\prime}(r)-l_{2}(r) f(r)=0, \quad r=b, \\
& r^{2} f^{\prime \prime}(r)+r f^{\prime}(r)+\left(k^{2} r^{2}-1\right) f(r)=0, \quad r=b,
\end{aligned}
$$

where $b=1 / \sqrt{\lambda}$, the differential operator $\triangle_{q}$ is defined by

$$
\triangle_{q}=r^{2} \frac{d^{2}}{d r^{2}}+r \frac{d}{d r}-\left(1+k^{2} q^{2} r^{2}\right)
$$

and

$$
\begin{aligned}
& q_{1}^{2}, q_{2}^{2}=\frac{\lambda^{4}+\lambda+\left(2 \lambda^{6}-4 \lambda^{3}+2\right) \omega_{1} \mp \sqrt{\left(\lambda^{4}+\lambda+2\left(\lambda^{3}-1\right)^{2} \omega_{1}\right)^{2}-4 \lambda^{5}}}{2 \lambda}, \\
& l_{1}(r)=\frac{2 k^{2}\left(\lambda^{3}-1\right)^{2} r^{2} \omega_{1}}{\lambda}+k^{2}\left(\lambda^{3}+2\right) r^{2}+1, \\
& l_{2}(r)=\frac{\gamma k^{2} \lambda r\left(k^{2} r^{2}-1\right)}{2 w_{d}}+\frac{2 k^{2}\left(\lambda^{3}-1\right)^{2} r^{2} \omega_{1}}{\lambda}+k^{2} \lambda^{3} r^{2}-1 .
\end{aligned}
$$

In the above expressions, $\omega_{1}=w_{d d} / w_{d}, w_{d}=w^{\prime}\left(2 \lambda^{-1}+\lambda^{2}\right)$, and $w_{d d}=w^{\prime \prime}\left(2 \lambda^{-1}+\lambda^{2}\right)$. The $q_{1}^{2}$ and $q_{2}^{2}$ correspond to the minus and plus choices in (3.6), respectively.

As shown by Wilkes (1955), a general solution of (3.2) that is bounded at $r=0$ is given by

$$
f(r)=\frac{I_{1}\left(k r q_{1}\right)}{I_{1}\left(k r_{0} q_{1}\right)}+c_{1} \frac{I_{1}\left(k r q_{2}\right)}{I_{1}\left(k r_{0} q_{2}\right)},
$$


where $I_{1}(x)$ denotes the Modified Bessel function of the first kind and $c_{1}$ is an arbitrary constant (see details in Goriely et al. (2008)). On substituting this solution into the second boundary condition (3.4), we obtain

$$
c_{1}=-\frac{1+q_{1}^{2}}{1+q_{2}^{2}}
$$

We note that the boundary condition (3.4) and hence this relation are both independent of the strain-energy function used. On substituting (3.9) and (3.10) into the first boundary condition, we obtain the bifurcation condition satisfied by $k, \lambda$ and $\gamma$. In Fig. 2, we show this bifurcation condition for two typical scenarios where either $\gamma$ or $\lambda$ is chosen as the control parameter. We see that in both cases, the minimum load occurs at $k_{\mathrm{cr}}=0$ for a critical $\gamma_{\text {cr }}$ or $\lambda_{\text {cr }}$. This is an important observation. As the bifurcation takes place, what modes do we expect to observe for $\gamma \gtrsim \gamma_{\mathrm{cr}}$ or $\lambda \gtrsim \lambda_{\mathrm{cr}}$ ? If the critical wavenumber $k_{\mathrm{cr}}$ was strictly positive, the emerging pattern would typically have a wavelength $1 / k_{\mathrm{cr}}$. But, this result is obviously not valid when $k_{\mathrm{cr}}=0$ and the linear analysis fails to give information on new solutions appearing close to the bifurcation. In that case, the general theory of dynamical systems and normal forms suggests that in such case the bifurcation may support a localized inhomogeneous solution at this critical load (Kirchgässner, 1982; Iooss \& Adelmeyer, 1999). Great care is needed here and our nonlinear near-critical analysis presented in the next section will study this problem in details and demonstrate explicitly that such a bifurcation does indeed take place, and that both bulging and necking solutions are possible.
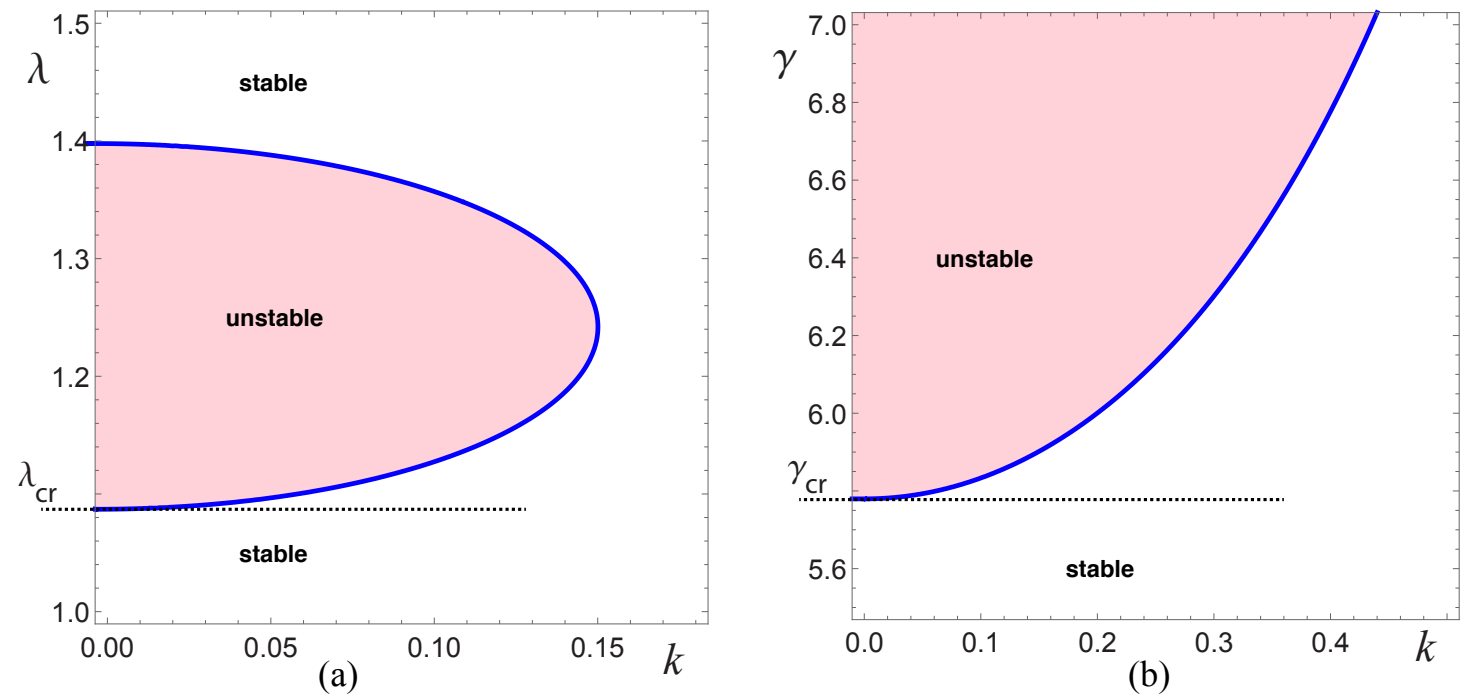

Figure 2: Bifurcation condition showing the dependence of (a) $\lambda$ on $k$ when $\gamma=5.8$ and (b) $\gamma$ on $k$ when $\lambda=1.1$. Both curves correspond to the Gent material model with $J_{m}=100$.

The above-mentioned minimum load can be determined analytically. Taking the limit $k \rightarrow 0$ in the bifurcation condition, we obtain $\gamma=\gamma_{\text {cr }}$ where

$$
\gamma_{\mathrm{cr}}=\frac{4 \lambda\left(\lambda^{3}+2\right) w_{d}+8\left(\lambda^{3}-1\right)^{2} w_{\mathrm{dd}}}{\lambda^{5 / 2}} .
$$

For the Gent model, we obtain

$$
\gamma_{\mathrm{cr}}=\frac{2 J_{m}\left(\lambda^{4} J_{m}+2 \lambda J_{m}+\lambda^{6}+3 \lambda^{4}-8 \lambda^{3}+6 \lambda-2\right)}{\sqrt{\lambda}\left(-\lambda J_{m}+\lambda^{3}-3 \lambda+2\right)^{2}} .
$$


This recovers, in the neo-Hookean limit $J_{m} \rightarrow \infty$, the well-known result (Taffetani \& Ciarletta, 2015)

$$
\gamma_{\mathrm{cr}}=\frac{2\left(2+\lambda^{3}\right)}{\lambda^{3 / 2}}
$$

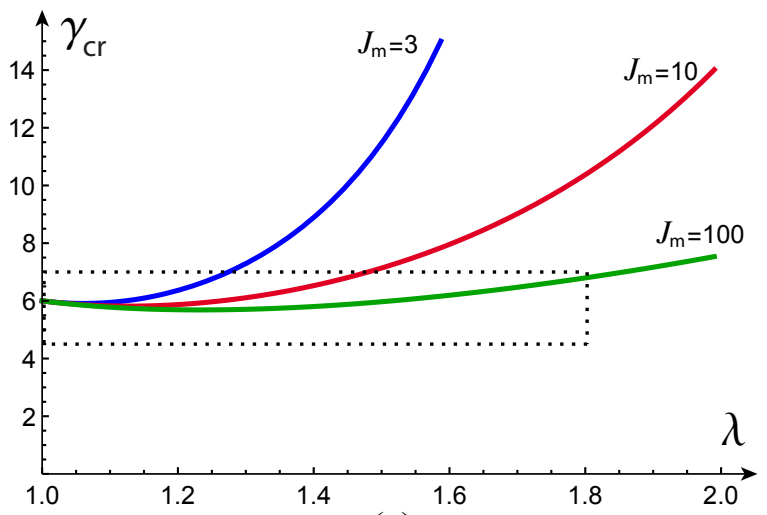

(a)

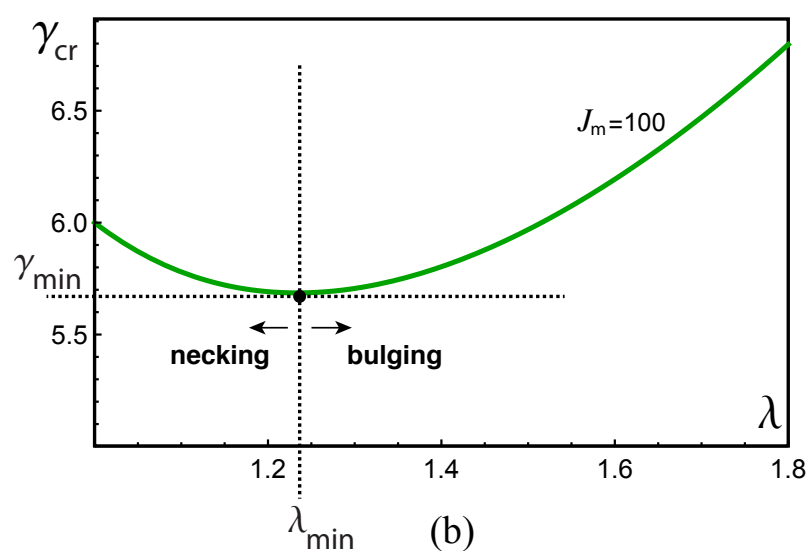

(b)

Figure 3: Dependence of $\gamma_{\mathrm{cr}}$ on $\lambda$ corresponding to the Gent material model. (a) $J_{m}=3,10,100$. (b) Blow-up of the dashed region in (a) for $J_{m}=100$ showing the existence of a minimum value of $\gamma_{\mathrm{cr}}$ over all values of $\lambda$

Fig. 3(a) shows the dependence of $\gamma_{\text {cr }}$ on the fixed axial stretch $\lambda$ for three representative values of $J_{m}$. We see that for each fixed axial stretch, smaller values of $J_{m}$ lead to larger values of $\gamma_{\mathrm{cr}}$ : a reduction in extensibility (smaller values of $J_{m}$ ) stabilizes against bulging and necking. Fig. 3(b) shows a blown-up of the behavior of $\gamma_{\mathrm{cr}}$ near $\lambda=1$. We observe that the critical value of $\gamma$ as a function of $\lambda$ has a minimum denoted by $\left(\lambda_{\min }, \gamma_{\min }\right)$. This property of the critical curve is true for all $J_{m}>0$, with asymptotic limit $\lambda_{\min } \rightarrow \sqrt[3]{2} \approx 1.26$ as $J_{m} \rightarrow \infty$, as shown in Fig. 4 . We will show that the existence of this minimum is an important feature of the problem as it divides possible behavior between bulging and necking.

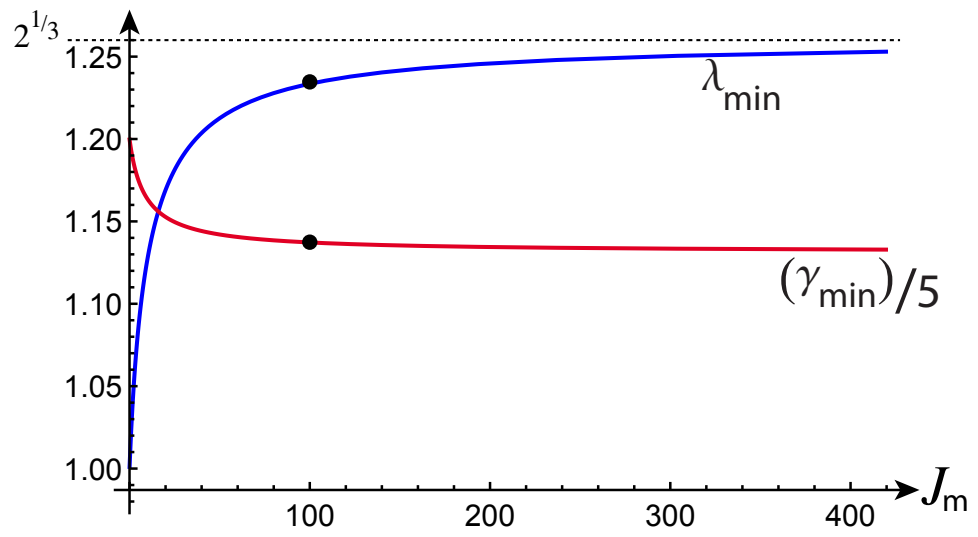

Figure 4: Dependence on $J_{m}$ of the minimum value of $\gamma_{\min }$ and the value of $\lambda_{\min }$ where it is attained. The dot corresponds to the values of Fig. 3 and the curve $\lambda$ tends to the neo-Hookean value of $\sqrt[3]{2}$ as shown in Fig. 5 . 


\subsection{Stress formulation}

The conditions that we have obtained so far for the bifurcation are in terms of the strains. An alternative derivation can be obtained by considering the stresses in the system following a similar derivation for the problem of localized bulging in inflated hyperelastic tubes in Fu et al. (2016). Let $N$ be the resultant axial force. We show now that the bifurcation condition (3.11) corresponds to an extremum of the curve $N=N(\lambda)$ at fixed $\gamma$ or an extremum of the curve $\gamma=\gamma(\lambda)$ at fixed $N$. We first note that the Cauchy stress is given by

$$
\sigma=2 w^{\prime}\left(\hat{I}_{1}\right) \mathbf{F} \mathbf{F}^{T}-p I
$$

where $p$ is the Lagrange multiplier associated with the incompressibility constraint. For the uniform deformation, application of the boundary condition $\sigma_{r r}=-\gamma / b$ at the outer surface $r=b$ yields

$$
p=\frac{2 w_{d}}{\lambda}+\frac{\gamma}{b}
$$

The Cauchy stress in the axial direction is then given by

$$
\sigma_{z z}=2\left(\lambda^{2}-\lambda^{-1}\right) w_{d}-\frac{\gamma}{b}
$$

The resultant axial force $N$ is computed according to $N=\pi b^{2} \sigma_{z z}+2 \pi b \gamma$ and is given by

$$
N=\pi\left(2\left(\lambda-\lambda^{-2}\right) w_{d}+\frac{\gamma}{\sqrt{\lambda}}\right) .
$$

It is then straightforward to verify that $d N / d \lambda=0$ yields (3.11) if $\gamma$ is fixed in the differentiation. Likewise, if (3.17) is solved to express $\gamma$ in terms of $N$ and $\lambda$, then $d \gamma / d \lambda=0$ with $N$ fixed yields

$$
N=2 \pi \frac{3 \lambda^{4} w_{d}+3 \lambda w_{d}+4 \lambda^{6} w_{d d}-8 \lambda^{3} w_{d d}+4 w_{d d}}{\lambda^{3}} .
$$

Elimination of $N$ from (3.17) and (3.18) gives (3.11).

In Fig. 5, we show the variation of $N$ with respect to $\lambda$ when $\gamma$ is fixed and variation of $\gamma$ when $N$ is fixed. We will show next that each extremum corresponds to a bifurcation into a localized solution.

\section{Weakly nonlinear near-critical analysis}

The linear bifurcation analysis presented in the previous section suggests that a bifurcation into a localized inhomogeneous solution is possible. The problem is now to find the bifurcated solution explicitly. Here, we use the methods of multiple scales and near-critical nonlinear analysis to explore the bifurcation, construct the localized solution, and analyze its properties.

\subsection{Taking $\gamma$ as the control parameter at fixed stretch $\lambda$}

We first take $\gamma$ to be our control parameter and assume that the axial stretch $\lambda$ is fixed. This represents the case when the cylinder is first subject to a specified axial stretch $\lambda$ and then constrained so that no more motion of the ends is possible. We note that previous studies have only considered the case when $\lambda=1$. As $\gamma$ is varied, the axial force $N$ has to be adjusted to maintain this constant stretch. We consider a material with the linear instability property depicted in Fig. 3(b) with the existence of a minimum for the critical curve, but we assume that the fixed stretch $\lambda \neq \lambda_{\min }$. The special case with $\lambda=\lambda_{\min }$ will be discussed separately. We note that varying 


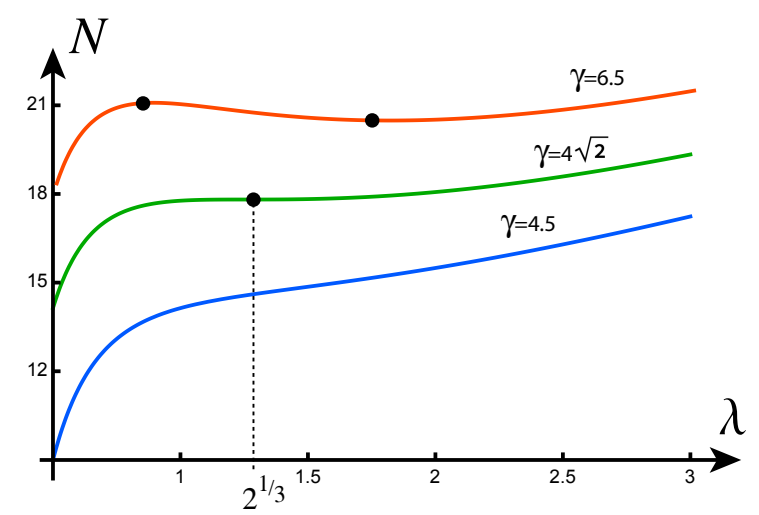

(a)

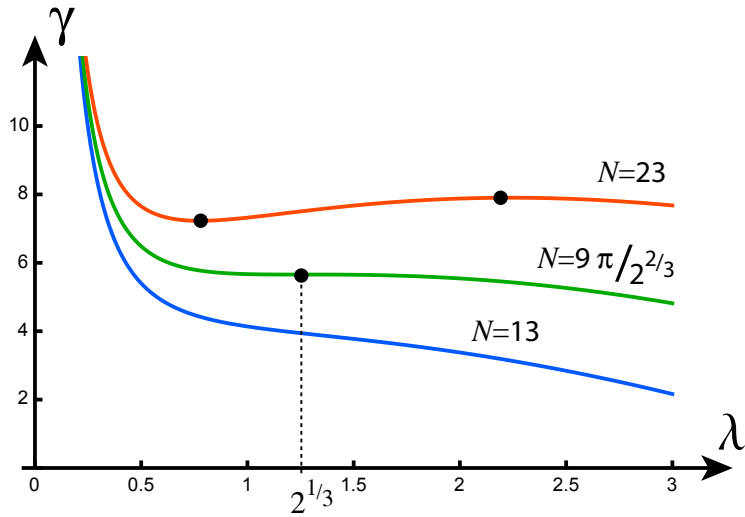

(b)

Figure 5: Bifurcation condition for a neo-Hookean material. (a) Variation of $N$ with respect to $\lambda$ for different values of $\gamma$. Two extrema are found for $\gamma>4 \sqrt{2} \approx 5.66$. At this value, the critical stretch is $\sqrt[3]{2}$. (b) Variation of $\gamma$ with respect to $\lambda$ for different values of dead-load $N$. A minimum exists only if $N>9 \pi / 2^{2 / 3} \approx 17.81$. For $N=9 \pi / 2^{2 / 3}$, the minimum occurs for $\lambda=\sqrt[3]{2}$.

the surface tension is not easy to achieve experimentally. It can be done, to a certain extent, by varying the temperature of chemical composition of the material. Here, we are mostly interested in the mathematical structure of the problem arising at the bifurcation as it will be similar to other, more realistic cases.

The main idea in a near-critical nonlinear analysis is to use the distance to the bifurcation as a small parameter. Therefore, we set

$$
\gamma=\gamma_{\mathrm{cr}}+\varepsilon \gamma_{1},
$$

where $\gamma_{\mathrm{cr}}$ is given by (3.11), $\gamma_{1}$ is a constant, and $\varepsilon$ is a small positive parameter. In addition, the linear analysis suggests the introduction of a far-distance variable $s$ through $(\mathrm{Fu}, 2001)$

$$
s=\sqrt{\varepsilon} z .
$$

We extend (3.1) and look for an asymptotic solution of the form

$$
\phi=\frac{z R^{2}}{2 \lambda}+\sqrt{\varepsilon}\left\{u^{(1)}(R, s)+\varepsilon u^{(2)}(R, s)+\varepsilon^{2} u^{(3)}(R, s)+\cdots\right\},
$$

where all the functions on the right-hand sides are to be determined from successive approximations. Corresponding to the above expansion, the variables $r$ and $Z$ given by (2.9) take the form

$$
r=\frac{R}{\sqrt{\lambda}}+\varepsilon \frac{\sqrt{\lambda}}{R}\left(u_{, s}^{(1)}+\varepsilon u_{, s}^{(2)}+\cdots\right), \quad Z=\frac{z}{\lambda}+\frac{\sqrt{\varepsilon}}{R}\left(u_{, R}^{(1)}+\varepsilon u_{, R}^{(2)}+\cdots\right) .
$$

On substituting (4.3) into (2.13)-(2.15) and equating the coefficients of like powers of $\varepsilon$, we obtain a hierarchy of boundary value problems. To leading order, we obtain the governing equation

$$
\mathcal{L}\left[u^{(1)}\right]=0
$$

and the boundary conditions

$$
\mathcal{B}^{(1)}\left[u^{(1)}\right]=0, \quad \mathcal{B}^{(2)}\left[u^{(1)}\right]=0, \quad R=1,
$$


where the three differential operators $\mathcal{L}, \mathcal{B}^{(1)}, \mathcal{B}^{(2)}$ are defined by

$$
\begin{aligned}
& \mathcal{L}[u]=u_{, R R R R}-\frac{2}{R} u_{, R R R}+\frac{3}{R^{2}} u_{, R R}-\frac{3}{R^{3}} u_{, R}, \\
& \mathcal{B}^{(1)}[u]=u_{, R R R}-\frac{1}{R} u_{, R R}+\frac{1}{R^{2}} u_{, R}, \\
& \mathcal{B}^{(2)}[u]=u_{, R R}-\frac{1}{R} u_{, R} .
\end{aligned}
$$

The general solution of (4.5) is given by

$$
u^{(1)}=C_{1}(s) R^{2}+C_{2}(s) R^{4}+C_{3}(s)\left[R^{2} \log (R)-\frac{1}{2} R^{2}\right]+C_{4}(s),
$$

where $C_{1}(s), \ldots, C_{4}(s)$ are unknown functions. It can be shown with the use of (4.4) that the incremental displacement is bounded at $R=0$ only if $C_{3}(s)=0$ and $C_{4}(s)$ is a constant. Since the shape $r=r(R, s)$ and $Z=Z(R, s)$ only depends on derivatives of $u^{(1)}$, we can set $C_{4}(s)=0$, without loss of generality, since as a constant it will not appear in any of the equations or boundary conditions. The same consideration will be applied at higher orders. The two boundary conditions in (4.6) require that $C_{2}(s)=0$. Thus, the leading-order solution reduces to

$$
u^{(1)}=C_{1}(s) R^{2} .
$$

At the next order, $u^{(2)}$ satisfies $\mathcal{L}\left[u^{(2)}\right]=0$ so that a similar argument to the one applied to $u^{(1)}$ gives

$$
u^{(2)}=D_{1}(s) R^{2}+D_{2}(s) R^{4},
$$

where the functions $D_{1}(s)$ and $D_{2}(s)$ are to be determined. The associated boundary conditions at this order are

$$
\begin{aligned}
& \mathcal{B}^{(1)}\left[u^{(2)}\right]=\frac{\gamma_{\mathrm{cr}} \lambda^{5 / 2}-4\left(\lambda^{4}+\lambda\right) w_{d}-8\left(\lambda^{3}-1\right)^{2} w_{\mathrm{dd}}}{2 \lambda^{2} w_{d}} C_{1}^{\prime \prime}(s), \quad R=1, \\
& \mathcal{B}^{(2)}\left[u^{(2)}\right]=\frac{1}{\lambda} C_{1}^{\prime \prime}(s), \quad R=1 .
\end{aligned}
$$

On substituting (4.12) into the (4.13) and (4.14), we obtain a homogeneous matrix equation of the form $M \boldsymbol{d}=\mathbf{0}$, where $\boldsymbol{d}=\left\{C_{1}^{\prime \prime}(s), D_{2}(s)\right\}^{T}$. For a non-trivial solution to exist, we must have $\operatorname{det}(M)=0$, which yields both the bifurcation condition (3.11) and the relation

$$
D_{2}(s)=\frac{\gamma_{\mathrm{cr}} \lambda^{5 / 2}-4\left(\lambda^{3}+1\right) \lambda w_{d}-8\left(\lambda^{3}-1\right)^{2} w_{d d}}{8 \lambda^{2} w_{d}} C_{1}^{\prime \prime}(s) .
$$

Finally, at third order, we find

$$
\mathcal{L}\left[u^{(3)}\right]=-\frac{2 \lambda^{4}+\lambda+2\left(\lambda^{3}-1\right)^{2} \omega_{1}}{\lambda^{3}} \cdot R^{2} C_{1}^{(4)}(s) .
$$

The general solution that is bounded at $R=0$ is given by

$$
u^{(3)}=G_{1}(s) R^{2}+G_{2}(s) R^{4}+\frac{1}{192} R^{6} C_{1}^{(4)}(s)\left(4 \omega_{1}-2 \lambda^{3} \omega_{1}-\frac{2 \omega_{1}}{\lambda^{3}}-\frac{1}{\lambda^{2}}-2 \lambda\right) .
$$

On substituting (4.17) into the two associated boundary conditions (which are not written out for the sake of brevity), we obtain a matrix equation of the form $\boldsymbol{M} \boldsymbol{g}=\boldsymbol{f}$, where $\boldsymbol{g}=\left\{D_{1}^{\prime \prime}(s), G_{2}(s)\right\}^{T}$, 
$M$ is the same singular matrix that appeared at second order, and the inhomogeneous term $\boldsymbol{f}$ only involves $C_{1}^{(4)}(s)$. The Fredholm condition applied to this matrix equation then yields the desired amplitude equation. After integrating this amplitude once and setting the arbitrary constant to zero for decay solutions, we obtain

$$
A^{\prime \prime}=k_{1} \gamma_{1} A+k_{2} A^{2}
$$

where $A(s)=C_{1}^{\prime}(s)$, and the coefficients $k_{1}$ and $k_{2}$ are given by

$$
k_{1}=-\frac{2 \lambda^{7 / 2}}{3 \lambda\left(3 \lambda^{3}+5\right) w_{d}+16\left(\lambda^{3}-1\right)^{2} w_{d d}}, \quad k_{2}=\lambda^{2} k_{1} \frac{d \gamma_{\mathrm{cr}}}{d \lambda} .
$$

For the Gent material model, these expressions take the form

$$
k_{1}=-\frac{4 \lambda^{3 / 2}\left(-\lambda J_{m}+\lambda^{3}-3 \lambda+2\right)^{2}}{J_{m}\left(3\left(3 \lambda^{3}+5\right) \lambda J_{m}+7 \lambda^{6}+27 \lambda^{4}-65 \lambda^{3}+45 \lambda-14\right)}, \quad k_{2}=4 \lambda^{2} \frac{k_{21}}{k_{22}},
$$

where

$$
\begin{aligned}
k_{21}= & 6 \lambda\left(2 \lambda^{6}+3 \lambda^{4}-6 \lambda+1\right) J_{m}+3 \lambda^{2}\left(\lambda^{3}-2\right) J_{m}^{2} \\
& +\left(\lambda^{6}+3 \lambda^{5}+42 \lambda^{4}+40 \lambda^{3}+24 \lambda^{2}-6 \lambda+4\right)(\lambda-1)^{3}, \\
k_{22}= & \left(-\lambda J_{m}+\lambda^{3}-3 \lambda+2\right)\left(3\left(3 \lambda^{3}+5\right) \lambda J_{m}+7 \lambda^{6}+27 \lambda^{4}-65 \lambda^{3}+45 \lambda-14\right) .
\end{aligned}
$$

Taking the limit $J_{m} \rightarrow \infty$, we obtain the following expressions for the neo-Hookean material model:

$$
k_{1}=-\frac{4 \lambda^{5 / 2}}{3\left(5+3 \lambda^{3}\right)}, \quad k_{2}=\frac{4 \lambda^{2}\left(2-\lambda^{3}\right)}{\left(5+3 \lambda^{3}\right)} .
$$

We note from $(4.23)_{2}$ that $k_{2} \rightarrow 0$ as $\lambda \rightarrow \sqrt[3]{2}=\lambda_{\text {min }}$, which signals the fact that this limit needs to be treated separately.

\subsubsection{Analysis of the amplitude equation}

The amplitude equation (4.18) is second-order and contains quadratic nonlinearity. A solution of this equation, after scaling back to the original variables, gives the radius

$$
r(R, z)=\frac{R}{\sqrt{\lambda}}(1+\varepsilon \lambda A(\sqrt{\varepsilon} z)+\cdots) .
$$

Depending on the sign of the coefficients $k_{1}$ and $k_{2}$, different solutions are possible. We note that, in general, $k_{1}<0$ and the sign of $k_{2}$ will change at the point where $\gamma_{\text {cr }}$ attains its minimum; see Fig. 3 and equation $(4.19)_{2}$. The amplitude equation can be written as a one degree-of-freedom Hamiltonian system

$$
A^{\prime \prime}=-\frac{\partial V}{\partial A}, \quad \text { with } \quad V=-\frac{k_{1} \gamma_{1}}{2} A^{2}-\frac{k_{2}}{3} A^{3} .
$$

For this system to support bounded solutions connecting asymptotically to the trivial solution, we must have $k_{1} \gamma_{1}>0$, which leads to two possibilities, as shown in Fig. 6. In particular, this system supports a localized homoclinic solution given by

$$
A(s)=-\frac{3 \gamma_{1} k_{1}}{2 k_{2}} \operatorname{sech}^{2}\left(\frac{1}{2} \sqrt{\gamma_{1} k_{1}} s\right)
$$


This solution also describes the shape of steady solitary wave solutions of the well-known Kortewegde Vries $(\mathrm{KdV})$ equation $u_{t}-6 u u_{x}+u_{x x x}=0$. By definition a negative value of $\gamma_{1}$ defines a value of surface tension below its critical value. Assuming that $\gamma_{1} k_{1}>0$, the bifurcation solution (4.26) is a bulge (a.k.a. a bright soliton) if $k_{2}<0$ or a depression (a.k.a. a dark soliton or necking) if $k_{2}>0$. From equation (4.23) we see that for the neo-Hookean model, the bifurcation is always subcritical, and the bifurcated solution represents a depression when $\lambda<\sqrt[3]{2}$ and a bulge when $\lambda>\sqrt[3]{2}$. This subcritical bifurcation is expected to be unstable and may not be directly observed as a static state (but could be observed dynamcally).

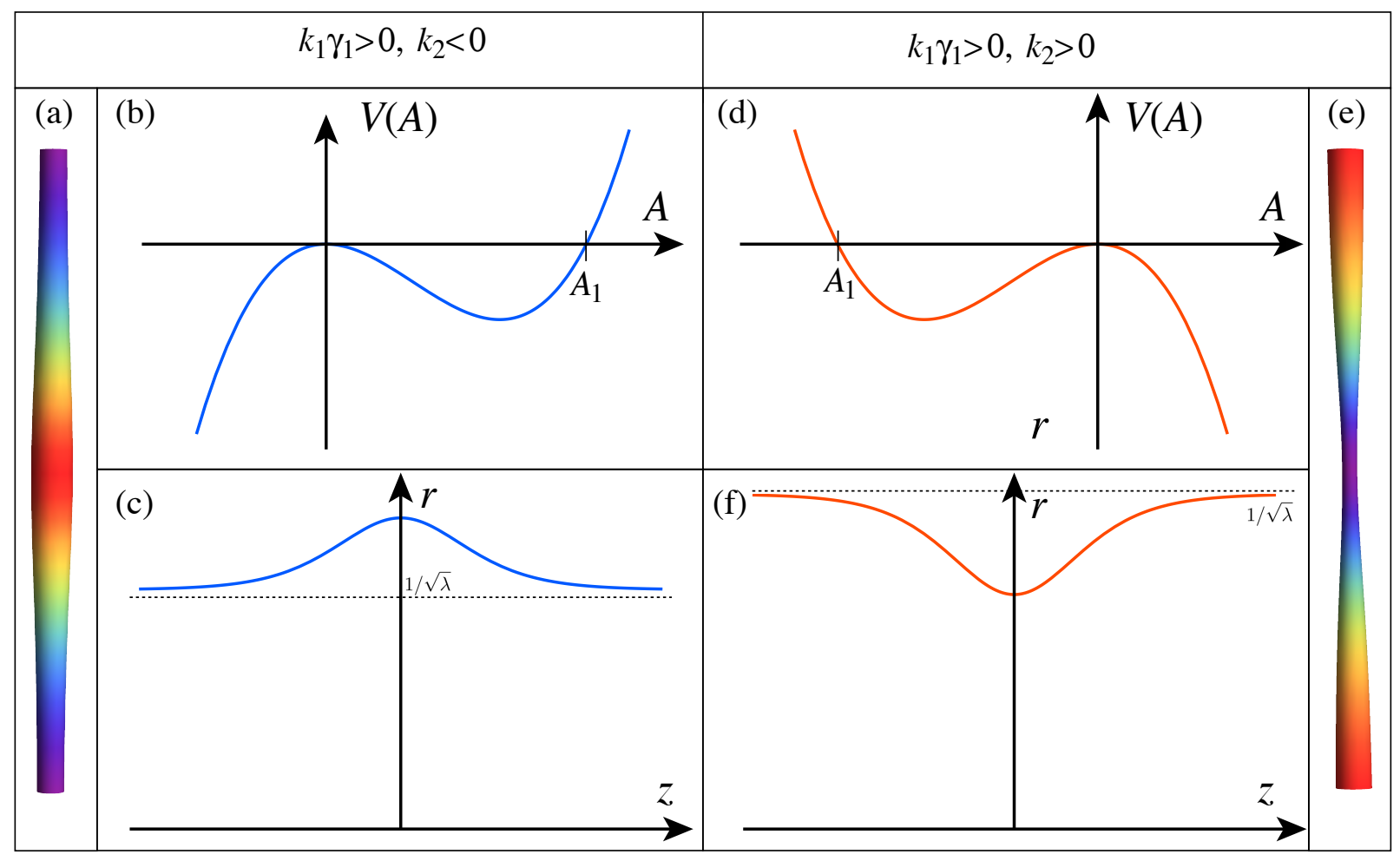

Figure 6: Bounded potentials (b,d) and localized homoclinic solutions (c,f) of the amplitude equations together with the corresponding cylindrical solutions giving a bulge (a) or a neck (e). The two different cases are given by the sign of $k_{2}$ and $A_{1}=-3 \gamma_{1} k_{1} /\left(2 k_{2}\right)$

Finally, we note that the homoclinic solutions are only valid on an infinite domain. For a long-enough finite cylinder, the system also supports periodic solutions that are given by periodic solutions of the amplitude equations with a period equal to $\sqrt{\varepsilon} \ell$. For long-enough tubes, these solutions are exponentially close to the homoclinic solutions and their qualitative behavior is fully captured by the simpler solution (4.26).

\subsubsection{The special turning point}

The localized solution (4.26) becomes invalid when $\lambda$ approaches the special value $\lambda_{\text {min }}$ (or equivalently $k_{2} \rightarrow 0$ ) where $\gamma$ attains its minimum as shown in Fig. 3(b). The form of (4.26) suggests that in the limit $\lambda \rightarrow \lambda_{\min }$, the far-distance variable $s$ remains valid, and it is the amplitude that should be re-scaled. Thus we look for an asymptotic solution of the form

$$
\phi=\frac{z R^{2}}{2 \lambda}+\hat{C}_{1}(s) R^{2}+\varepsilon^{1 / 2} v^{(1)}(R, s)+\varepsilon v^{(2)}(R, s)+\varepsilon^{3 / 2} v^{(3)}(R, s)+\varepsilon^{2} v^{(4)}(R, s)+\cdots,
$$


where all the functions on the right hand sides are to be determined from successive approximations, and the choice of the leading term of perturbation, i.e. $\hat{C}_{1}(s) R^{2}$, has been motivated by (4.11). We observe that although this leading term is of the same order as the first term representing the primary deformation, the associated deformation gradient is of order $\varepsilon^{1 / 2}$.

Since the $\gamma$ versus $\lambda$ curve in Figure 3(a) is locally parabolic near $\lambda=\lambda_{\min }$, we may also incorporate an order $\varepsilon^{1 / 2}$ variation of $\lambda$ around this minimum by writing

$$
\gamma=\gamma_{\min }+\varepsilon \hat{\gamma}_{1}, \quad \lambda=\lambda_{\min }+\varepsilon^{1 / 2} \hat{\lambda}_{1}
$$

where $\hat{\lambda}_{1}$ and $\hat{\gamma}_{1}$ are constants. For the sake of brevity, we only give an outline of our derivations. The order $\varepsilon^{1 / 2}$ solution $v^{(1)}(R, s)$ is found to be identically zero. At order $\varepsilon$, we obtain the bifurcation condition (3.11), whereas at order $\varepsilon^{3 / 2}$ we obtain $d \gamma_{\mathrm{cr}} / d \lambda=0$ at $\lambda=\lambda_{\min }$, that is satisfied automatically. The amplitude equation for $\hat{A}=\hat{C}_{1}^{\prime}(s)$ is obtained at order $\varepsilon^{2}$ as

$$
\begin{aligned}
\hat{A}^{\prime \prime}(s) & =\left(\hat{k}_{1} \hat{\gamma}_{1}+\hat{k}_{2} \hat{\lambda}_{1}^{2}\right) \hat{A}-2 \lambda_{\min }^{2} \hat{\lambda}_{1} \hat{k}_{2} \hat{A}^{2}+\frac{4}{3} \lambda_{\min }^{4} \hat{k}_{2} \hat{A}^{3}, \\
& =\hat{k}_{2} \hat{A}\left\{\frac{4}{3}\left(\lambda_{\min }^{2} \hat{A}-\frac{3}{4} \hat{\lambda}_{1}\right)^{2}+\frac{1}{4} \hat{\lambda}_{1}^{2}+\frac{\hat{k}_{1}}{\hat{k}_{2}} \hat{\gamma}_{1}\right\}
\end{aligned}
$$

where $\hat{k}_{1}$ is the $k_{1}$ given by (4.19) evaluated at $\lambda=\lambda_{\min }$, and

$$
\hat{k}_{2}=-\left.\frac{1}{2} \hat{k}_{1} \frac{d^{2} \gamma_{\mathrm{cr}}}{d \lambda^{2}}\right|_{\lambda=\lambda_{\min }} .
$$

In terms of the original variables, the radius in this parameter regime is given by

$$
r(z)=\frac{R}{\sqrt{\lambda_{\min }+\varepsilon^{1 / 2} \hat{\lambda}_{1}}}\left\{1+\sqrt{\varepsilon} \lambda_{\min } \hat{A}(\sqrt{\varepsilon} z)+\cdots\right\} .
$$

This expression should be compared with (4.24) for the non-degenerate case.

In the neo-Hookean case, we have $\hat{k}_{1} \approx-0.216, \hat{k}_{2} \approx 0.866$. Corresponding to the Gent material model, both $\hat{k}_{1}$ and $\hat{k}_{2}$ are monotonically decreasing functions of $J_{m}$, and $\hat{k}_{1}$ is negative whereas $\hat{k}_{2}$ is positive for all positive values of $J_{m}$. Hence, to fix ideas we assume $\hat{k}_{1}<0$ and $\hat{k}_{2}>0$ in our subsequent analysis. In the general case, necessary conditions for a localized solution for $\hat{A}$ connecting to 0 asymptotically are

$$
\hat{k}_{1} \hat{\gamma}_{1}+\hat{k}_{2} \hat{\lambda}_{1}^{2}>0, \quad \frac{1}{4} \hat{\lambda}_{1}^{2}+\frac{\hat{k}_{1}}{\hat{k}_{2}} \hat{\gamma}_{1} \leq 0 .
$$

The former ensures that the solution decays as $s \rightarrow \pm \infty$ (and is related to the linear term in (4.29)), whereas the latter ensures that in the phase plane there are fixed points other than $\hat{A}=0$ (see the last terms in (4.30)). Under the assumption $\hat{k}_{2}>0$ these conditions may be reduced to

$$
-\hat{k}_{1} \hat{\gamma}_{1}<\hat{k}_{2} \hat{\lambda}_{1}^{2} \quad \text { and } \quad \hat{k}_{2} \hat{\lambda}_{1}^{2} \leq-4 \hat{k}_{1} \hat{\gamma}_{1}
$$

In particular, if $\hat{\lambda}_{1}=0$, these inequalities cannot be satisfied, and no localized solutions exist. This explains the numerical result, to be presented later, that when $\gamma$ is increased above $\gamma_{\min }$ with $\lambda$ fixed at $\lambda_{\min }$, it is a "two-phase" deformation that will bifurcate from the homogeneous solution. If $\hat{\lambda}_{1} \neq 0$, we may assume without loss of generality $\hat{\lambda}_{1}>0$ since the solution for the other case of $\hat{\lambda}_{1}<0$ can then be obtained by changing the sign of $\hat{A}$ (then from (4.32) the solution changes 
character: from bulging to necking). The non-zero fixed points of the dynamical system (4.30) are given by

$$
\lambda_{\min }^{2} \hat{A}=\frac{3}{4} \hat{\lambda}_{1} \pm \frac{3}{4} \sqrt{\hat{\lambda}_{1}^{2}-\frac{4}{3}\left(\hat{\lambda}_{1}^{2}+\frac{\hat{k}_{1}}{\hat{k}_{2}} \hat{\gamma}_{1}\right)},
$$

and we denote them by $\hat{A}^{+}$and $\hat{A}^{-}$with the superscripts corresponding to the \pm signs in (4.35), respectively. The assumptions (4.34) 1 ensure that both fixed-points are positive. It can be further verified that $\hat{A}^{+}$is a saddle whereas $\hat{A}^{-}$is a center; see Fig. 7(a). It then follows that a homoclinic orbit, and hence a localized solution, can exist, and this homoclinic orbit may also degenerate into a heteroclinic orbit when it connects to the fixed point $\hat{A}^{+}$(this occurs when $\hat{B}^{+}$coalesces with $\hat{B}^{-}$). The homoclinic solutions close to this heteroclinic solution are known as kink-wave solutions.

To obtain an explicit expression for the localized and kink-wave solutions, we write (4.30) as a one degree-of-freedom Hamiltonian system

$$
\hat{A}^{\prime \prime}=-\frac{\partial V}{\partial \hat{A}}, \quad \text { with } \quad V=-\frac{1}{3} \lambda_{\min }^{4} \hat{k}_{2} \hat{A}^{2}\left(\hat{A}-B^{+}\right)\left(\hat{A}-B^{-}\right)
$$

where the zeroes of the potential $B^{ \pm}$are given by

$$
\lambda_{\min }^{2} B^{ \pm}=\hat{\lambda}_{1} \pm \sqrt{-\frac{1}{2 \hat{k}_{2}}\left(3 \hat{k}_{1} \hat{\gamma}_{1}+\hat{k}_{2} \hat{\lambda}_{1}^{2}\right)},
$$

and we have neglected the arbitrary constant to satisfy the condition that $\hat{A}$ must approach the trivial solution when $s$ tends to plus or minus infinity. The expression under the radical sign in (4.37) is positive only if $\hat{k}_{2} \hat{\lambda}_{1}^{2} \leq-3 \hat{k}_{1} \hat{\gamma}_{1}$ which, together with (4.34), implies that the localized solution only exist for

$$
\lambda_{1}^{*}<\left|\hat{\lambda}_{1}\right|<\sqrt{3} \lambda_{1}^{*}, \quad \text { where } \quad \lambda_{1}^{*}=\sqrt{-\hat{k}_{1} \hat{\gamma}_{1} / \hat{k}_{2}}
$$

See Fig. 7 for the possible shapes of the potential and the position of these values in the parameter plane.
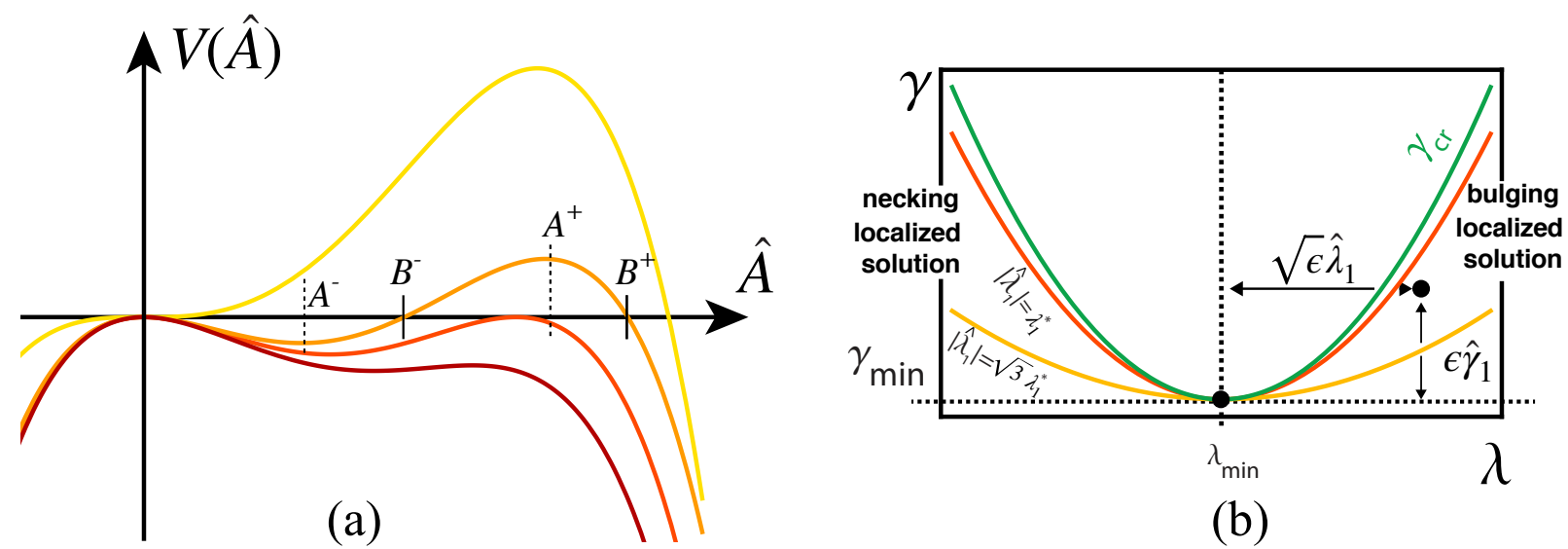

(b)

Figure 7: (a) Possible potentials $V(A)$. Only one of these potentials support a localized solution (connecting the origin to $B^{-}$orange). In the limit $B^{-} \rightarrow B^{+}$, the localized solution degenerates into a heteroclinic solution (red). (b) Domain of existence of a localized solution in the $\gamma-\lambda$ plane. For a solution to exist close to $\lambda=\lambda_{\min }$, the value of $\lambda$ must lie in either strips $\lambda_{1}^{*}<\left|\hat{\lambda}_{1}\right|<\sqrt{3} \lambda_{1}^{*}$. 
In this interval, the amplitude equation (4.36) has a localized solution given by

$$
\hat{A}=\frac{B^{+} B^{-}\left(1-\xi^{2}\right)}{B^{+}-B^{-} \xi^{2}}, \quad \text { where } \xi=\tanh \left[-\sqrt{\frac{\hat{k}_{2} B^{+} B^{-}}{6}} \lambda_{\min }^{2} s\right] .
$$

In the limit $B^{+} \rightarrow B^{-}$, that is when $\hat{\lambda}_{1} \rightarrow \sqrt{3} \lambda_{1}^{*}$, the above localized solution becomes a heteroclinic (aka kink-wave) solution that tends to 0 and $B^{+}$as $s \rightarrow \pm \infty$, respectively. In Fig. 8(a, b) we have shown the evolution of the solution given by (4.39) as $\hat{\lambda}_{1} \rightarrow \sqrt{3} \lambda_{1}^{*}$ or $\hat{\lambda}_{1} \rightarrow \lambda_{1}^{*}$, respectively. The presented results correspond to the neo-Hookean material model. It is seen from Fig. 8(a) that as $\hat{\lambda}_{1}-3 \lambda_{1}^{*}$ is reduced the localized solution becomes flatter and flatter at $s=0$, whereas Fig. 8(b) shows that as $\hat{\lambda}_{1}-\lambda_{1}^{*}$ is reduced, the amplitude of the solitary wave solution goes to zero rapidly and the solution spreads over a larger and larger interval. Note also that the expression (4.38) is real only if $\hat{k}_{1} \hat{\gamma}_{1} / \hat{k}_{2}<0$. Thus for neo-Hookean materials, $\hat{\gamma}_{1}$ must necessarily be positive. However, in the region where localized solutions exist (see Fig. $7(\mathrm{~b})$ ), we have $\gamma<\gamma_{\mathrm{cr}}$ and the bifurcation is subcritical.

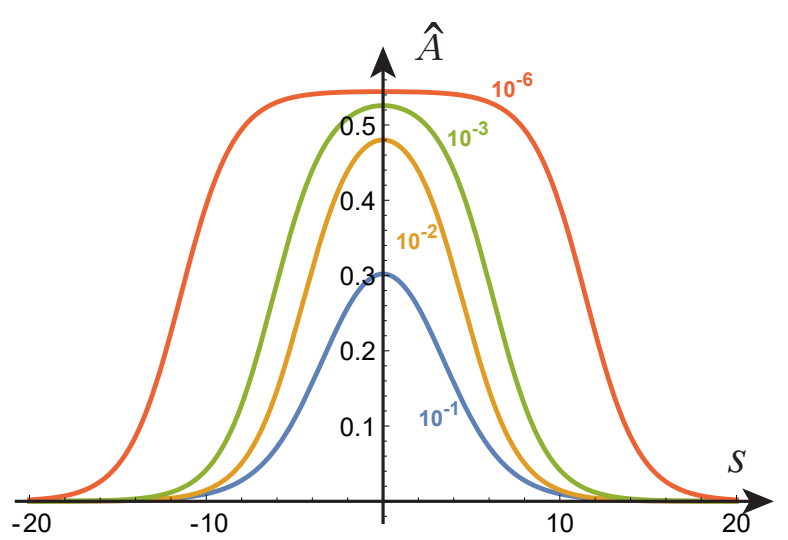

(a)

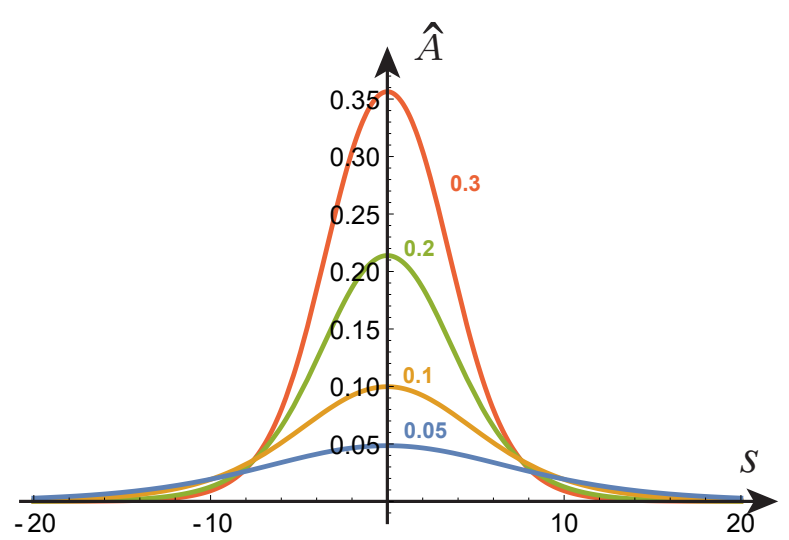

(b)

Figure 8: (a) Evolution of the solution given by (4.39) as $\hat{\lambda}_{1}-\sqrt{3} \lambda_{1}^{*}$ approaches zero. The four profiles correspond to $\hat{\lambda}_{1}-\sqrt{3} \lambda_{1}^{*}=10^{-1}, 10^{-2}, 10^{-3}, 10^{-6}$, respectively; the smaller the $\hat{\lambda}_{1}-\sqrt{3} \lambda_{1}^{*}$ the flatter the curve. (b) Evolution of the solution given by (4.39) as $\lambda_{1}^{*}-\hat{\lambda}_{1}$ tends to zero. The four curves corresponds to $\hat{\lambda}_{1}-\lambda_{1}^{*}=0.3,0.2,0.1,0.05$, respectively; smaller values of $\hat{\lambda}_{1}-\lambda_{1}^{*}$ correspond to smaller amplitude.

We may now collect our results and summarize the evolution of the localized solutions with respect to $\lambda$ as follows. The variation of the localized solution with respect to $\lambda$ has a boundary layer structure around $\lambda=\lambda_{\min }$. In an $O(\sqrt{\varepsilon})$ boundary layer centered at $\lambda_{\min }$, the amplitude of solution is of order $\sqrt{\varepsilon}$ and the bifurcation is subcritical (with $\hat{\gamma}_{1}>0$ but $\gamma<\gamma_{\text {cr }}$ ); outside this boundary layer the amplitude of solution is of order $\varepsilon$ and the bifurcation is also subcritical (with $\left.\hat{\gamma}_{1}<0\right)$. Within the boundary layer, the localized solution only exists for $\lambda_{1}^{*}<\left|\hat{\lambda}_{1}\right|<\sqrt{3} \lambda_{1}^{*}$, that is

$$
-\frac{\hat{k}_{2}}{3 \hat{k}_{1}} \cdot\left(\lambda-\lambda_{\min }\right)^{2}<\gamma-\gamma_{\min }<-\frac{\hat{k}_{2}}{\hat{k}_{1}} \cdot\left(\lambda-\lambda_{\min }\right)^{2} .
$$

At the left end of this interval, the homoclinic localized solution tends a heteroclinic kink-wave solution and therefore exhibits a long plateau. At the right end of the interval, the amplitude of the localized solution tends to zero while spreading over a larger and larger interval. Note that the upper boundary $\left|\hat{\lambda}_{1}\right|=\lambda_{1}^{*}$ in Fig. 7(b) for the existence of localized solutions is below the 
curve $\gamma=\gamma_{\text {cr }}$ given by the linear theory. As a result, as $\gamma$ is increased from 0 at fixed $\lambda$ (in a small neighbourhood of $\lambda_{\text {cr }}$ ), localization will initiate slightly earlier than predicted by the linear theory, and due to the small gap between the upper and lower boundaries in Fig. 7(b) the value of $\gamma$ at which the localized bulge or depression starts to propagate is only marginally smaller than the value at which localization initiates. The latter fact is indeed observed in our numerical simulations; see discussions related to Fig. 10 later. Finally, any localized solution corresponding to $\lambda<\lambda_{\min }$ represents necking, whereas any localized solution with $\lambda>\lambda_{\min }$ corresponds to bulging.

We conclude this section by observing that the heteroclinic solution mentioned below equation (4.39) has previously been characterized by Xuan \& Biggins (2017). By taking the limit $\hat{\lambda}_{1} \rightarrow \sqrt{3} \lambda_{1}^{*}$ and specializing to the neo-Hookean material, followed by an appropriate substitution of variables, we have verified that our amplitude equation (4.30) can recover their equation (A34) provided their variable $\tilde{Z}$ is identified with $\sqrt{\varepsilon} Z / a$ (their definition $\tilde{Z}=\sqrt{\varepsilon} z / a$ seems to be a typo).

\subsection{Taking $\lambda$ as the bifurcation parameter with fixed $\gamma$}

A second loading scenario that may be of interest is when surface tension $\gamma$ is specified and the axial force $N$ is taken to be the load parameter. Experimentally, this loading is easier to achieve as it is equivalent to taking the axial stretch $\lambda$ to be the load parameter since $N$ is a monotonically increasing function of $\lambda$ up to the point of bifurcation. Referring to Figure 5(a), we anticipate that a bifurcation takes place when the axial force reaches the maximum.

We write

$$
\lambda=\lambda_{\mathrm{cr}}+\varepsilon \lambda_{1},
$$

where $\lambda_{\text {cr }}$ is related to $\gamma$ by

$$
\gamma=\left.\frac{4 \lambda\left(\lambda^{3}+2\right) w_{d}+8\left(\lambda^{3}-1\right)^{2} w_{\mathrm{dd}}}{\lambda^{5 / 2}}\right|_{\lambda=\lambda_{\text {cr }}},
$$

which is adapted from (3.11), and $\lambda_{1}$ is a constant. We use the same stretched variable $s$ and the same form of asymptotic solution as in Section 4.1.1. Then, the same step-by-step analysis leads to the amplitude equation

$$
A^{\prime \prime}(s)=\tilde{k}_{1} \lambda_{1} A(s)+\tilde{k}_{2} A^{2}(s),
$$

where $\tilde{k}_{2}$ is the same as the $k_{2}$ given by $(4.19)_{2}$ except that $\lambda$ is replaced by $\lambda_{\text {cr }}$, and the linear coefficient $\tilde{k}_{1}$ is given by

$$
\tilde{k}_{1}=-\tilde{k}_{2} / \lambda_{\mathrm{cr}}^{2}
$$

This special dependence between the two coefficients can be explained as follows. To leading order in $\varepsilon$, the principal stretch in the axial direction is given by

$$
\lambda_{\mathrm{cr}}+\varepsilon \lambda_{1}-2 \varepsilon \lambda_{\mathrm{cr}}^{2} A(s) .
$$

Since the axial force $N$ attains its maximum at $\lambda=\lambda_{\text {cr }}$, the local behavior of $N$ versus $\lambda$ in a small neighborhood of $\lambda=\lambda_{\text {cr }}$ must be parabolic. This mean that if $A(s)$ is a constant the above principal stretch and the stretch $\lambda_{\mathrm{cr}}+\varepsilon \lambda_{1}$ must lie on opposite sides of, and be equidistance from, $\lambda=\lambda_{\mathrm{cr}}$, that is,

$$
\lambda_{\mathrm{cr}}-\left(\lambda_{\mathrm{cr}}+\varepsilon \lambda_{1}-2 \varepsilon \lambda_{\mathrm{cr}}^{2} A(s)\right)=\varepsilon \lambda_{1} .
$$

Hence, the homogeneous solution $A(s)=\lambda_{1} / \lambda_{\text {cr }}^{2}$ must be a possible solution of the amplitude equation (4.43), from which the above relation between $\tilde{k}_{2}$ and $\tilde{k}_{1}$ must necessarily follow. We also note the connection

$$
\left.\frac{d^{2} N}{d \lambda^{2}}\right|_{\text {fixed } \gamma}=-\frac{\pi w_{d}\left(16\left(\lambda_{\mathrm{cr}}^{3}-1\right)^{2} \omega_{1}+3 \lambda_{\mathrm{cr}}\left(3 \lambda_{\mathrm{cr}}^{3}+5\right)\right)}{4 \lambda_{\mathrm{cr}}^{7}} \tilde{k}_{2} .
$$


This means that $\tilde{k}_{2}$ vanishes when the first turning point in Fig.5(a) degenerates into an inflection point, as expected.

As an illustrative example, consider the neo-Hookean material model and take $\gamma=6.5$. There are two bifurcation values, $\lambda_{\mathrm{cr}}=0.879$ or 1.805 , corresponding to the maximum and minimum of $N$, respectively. The coefficients in the amplitude equation are given by

$$
\left(\tilde{k}_{1}, \tilde{k}_{2}\right)=(-0.750,0.580), \text { and }(0.686,-2.235)
$$

respectively. It then follows that the localized solution corresponding to the maximum is a depression whereas that corresponding to the minimum is a bulge.

\subsection{Taking $\gamma$ as the bifurcation/load parameter with $N$ fixed}

Finally we consider the loading scenario corresponding to Fig. 5(b). We fix the axial force $N$ and increase the surface tension in order to induce a bifurcation. This case is similar to the case studied in the previous subsection except now we need to eliminate $N$ in favor of $\gamma$ with the aid of (3.17). We observe that a positive $N$ at zero $\gamma$ will initially produce an axial stretch such that $\lambda>1$, and as $\gamma$ is increased from zero $\lambda$ decreases. Thus increasing $\gamma$ corresponds to traversing each curve in $5(\mathrm{~b})$ from right to the left, and when $N$ is large enough $\gamma$ first reaches a maximum and then a minimum. In a small neighborhood of the maximum or minimum, expressions (4.41) and the asymptotic expansion (4.3) are still valid. The same amplitude equation (4.43) with the same coefficients are found (in the previous subsection $\gamma$ is eliminated in the coefficients with the aid of the bifurcation condition (3.11), whereas here it is the $N$ that is eliminated). However, the interpretation is different. As an illustrative example, consider again the neo-Hookean material model and now take $N=6.5 \pi$. There are two bifurcation values, $\lambda_{\text {cr }}=1.885$ or 0.882 , corresponding to the maximum and minimum of $\gamma$, respectively. The coefficients in the amplitude equation are given by

$$
\left(\tilde{k}_{1}, \tilde{k}_{2}\right)=(0.749,-2.663), \text { or }(-0.744,0.579),
$$

respectively. We note that it is the maximum that is of interest here since it is reached first as $\gamma$ is increased from zero. The associated bifurcation is subcritical and corresponds to a bulge.

\section{Fully nonlinear post-bifurcation behavior and numerical simulations}

Our analysis so far has only captured the near-critical behavior. However, this problem has the same geometric and mathematical structure as the problem of localized bulging of inflated hyperelastic tubes. Therefore, we expect that much of the same post-bifurcation behaviors will also be similar. For instance, in the case of fixed surface tension and variable axial force, or fixed axial force and variable surface tension, we expect that the localization amplitude will grow until it has almost reached a maximum amplitude, after which the localization will propagate in the axial direction (the Maxwell state). We use the word "almost" because the maximum amplitude corresponds to a heteroclinic orbit which can only live over an infinite domain. The Maxwell state can be determined from Fig. 5 with the aid of the equal area rule. For instance, for the situation in Fig. 5(a), we may first use (3.17) to compute the integral

$$
G(\lambda) \equiv \int S_{z z} d \lambda=2 \gamma \sqrt{\lambda}+w\left(\lambda^{2}+2 \lambda^{-1}\right), \quad \text { where } S_{z z} \equiv N / \pi .
$$

The $S_{z z}$ defined above denotes the axial force per unit area in the reference configuration (i.e. the nominal stress). Then the Maxwell line intersects the load curve at $\lambda=\lambda_{1}, \lambda_{2}$ where

$$
S_{z z}\left(\lambda_{1}\right)=S_{z z}\left(\lambda_{2}\right), \quad S_{z z}\left(\lambda_{1}\right)\left(\lambda_{2}-\lambda_{1}\right)=G\left(\lambda_{2}\right)-G\left(\lambda_{1}\right) .
$$


Corresponding to the neo-Hookean model, the above two equations can be solved explicitly to yield

$$
\lambda_{2}=\frac{2^{2 / 3}}{\lambda_{1}}, \quad S_{z z}=\frac{3\left(\lambda_{1}^{2}+\sqrt[3]{2} \lambda_{1}+2^{2 / 3}\right)}{2 \lambda_{1}},
$$

and (5.46) then gives

$$
\gamma=\frac{\lambda_{1}^{3}+3 \lambda_{1}\left(\sqrt[3]{2} \lambda_{1}+2^{2 / 3}\right)+2}{2 \lambda_{1}^{3 / 2}},
$$

where the $\lambda_{1}$ in the expressions of $S_{z z}$ and $\gamma$ can be replaced by $\lambda_{2}$.

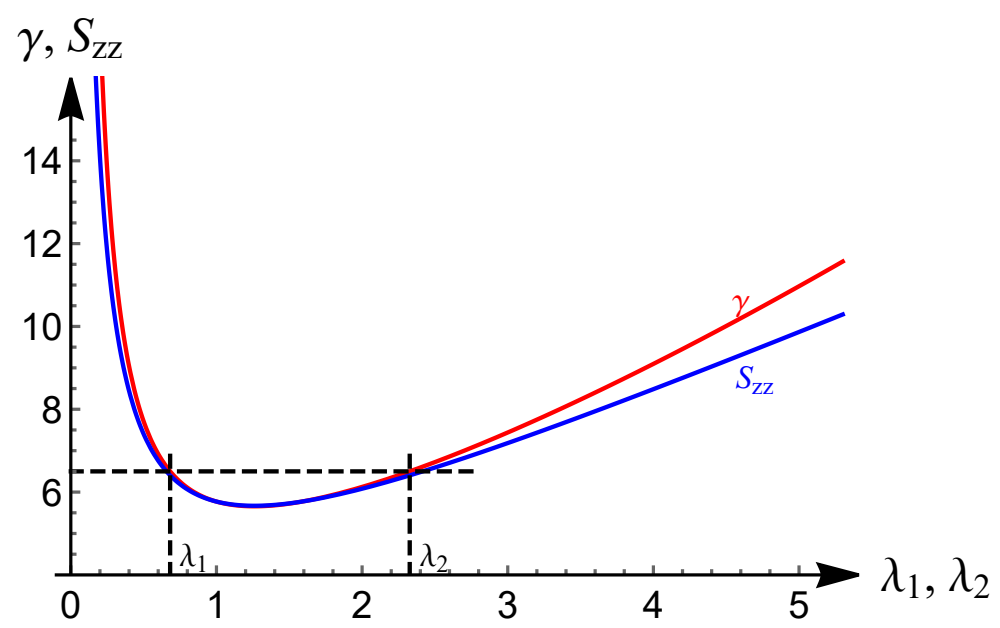

Figure 9: Plots of (5.48) 2 and (5.49). The left and right branches correspond, respectively, to $\lambda_{1}$ and $\lambda_{2}$ in the "two phases" when the localized depression has fully developed into a Maxwell state. For instance at $\gamma=6.5$, the Maxwell value of $S_{z z}$ is 6.403 , corresponding to $\lambda_{1}=0.682, \lambda_{2}=2.327=2^{2 / 3} / \lambda_{1}$. The minimum of $\gamma$ occurs at $\left(\lambda_{\min }, \gamma_{\min }\right)$.

Fig. 9 shows the dependence of $\gamma$ and $S_{z z}$ on $\lambda_{1}$ when the localized depression is fully developed into a Maxwell state. For each specified $\gamma$, the corresponding value of $\lambda_{1}$ can be read off from the left branch of the red solid curve in Fig. 9 or determined from (5.49). The corresponding $S_{z z}$ can then be computed from (5.48) $)_{2}$ as shown in Fig. 9. For later use, the Gent material model with $J_{m}=100$ and $\gamma=6.5$ gives values of $S_{z z}=6.417, \lambda_{1}=0.726, \lambda_{2}=2.148$.

To verify the theoretical predictions, we have conducted numerical simulations in Abaqus (Abaqus, 2013) by adapting the user subroutines of Henann \& Bertoldi (2014). In our simulations we adopted the same dimensions and material properties for the cylinder: $\mu=20 \mathrm{~Pa}, L=10 \mathrm{~mm}$, $B=0.25 \mathrm{~mm}$ (after this one-off use of dimensional quantities the $\mathrm{L}$ and $\mathrm{B}$ will again be scaled by $B$ ). All our simulations are conducted for the Gent material model with $J_{m}=100$. In Fig. 10 we have presented a typical set of results for the case when $\gamma$ is fixed at 6.5 and loading is carried out by varying $\bar{\lambda}$, the averaged axial stretch (i.e. the total deformed length divided by the undeformed length). This loading scenario corresponds to the situation in Fig. 5(a). The axial nominal stress $S_{z z}$ is initially zero with $\bar{\lambda}=\lambda=0.29$; the latter stretch is caused by the imposed surface tension that increases the radius from 1 to $1 / \sqrt{0.29} \approx 1.86$ (note that lengths are scaled by the undeformed radius). The $\bar{\lambda}$ is then increased from this initial value and the actual variation of $S_{z z}$ is simulated using Abaqus. According to Fig. 5(a) and our theory, a localized depression will initiate when $S_{z z}$ reaches the maximum 6.518 at $\bar{\lambda}=\lambda=0.881$ (corresponding to the left dot in Fig. 10), after which the amplitude of depression will increase until $r(1,0)$, the deformed radius at $Z=0$, has almost reached its minimum. This increase in the necking amplitude is accompanied 


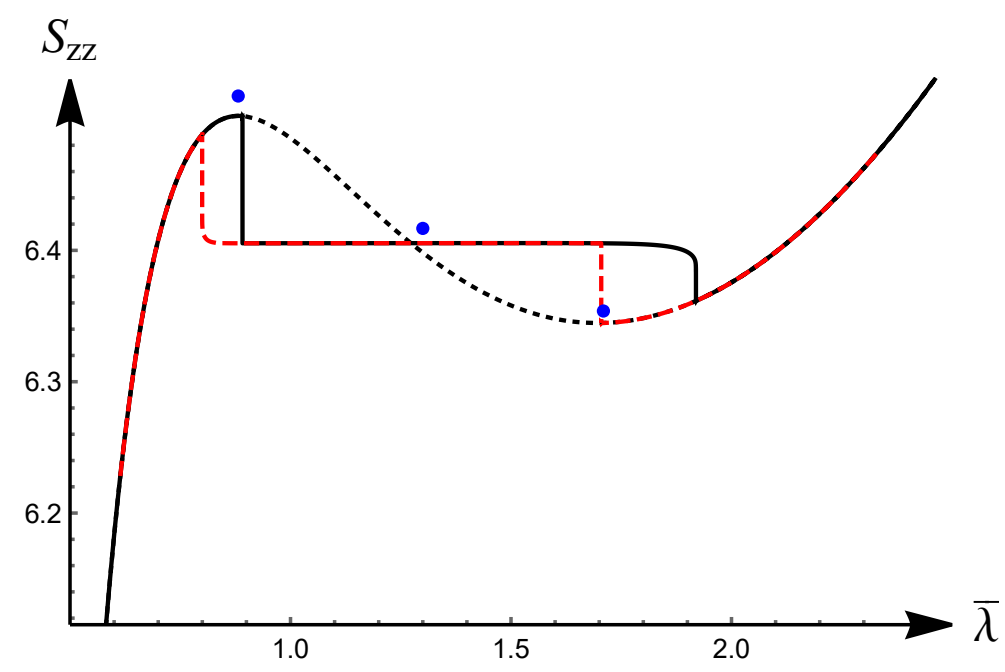

Figure 10: Comparison between theoretical predictions and Abaqus simulations. The solid and (red) dashed lines are simulation results corresponding to loading and unloading respectively. The left and right dots are the theoretical predictions for onset of necking (when loading) and bulging (when unloading), respectively, and the middle dot represents the theoretical prediction for the Maxwell value associated with the propagation stage. The (black) dotted line corresponds to $L=2$ and is included for comparison.

by unloading at the two ends and reduction of the axial nominal stress. As $\bar{\lambda}$ is further increased, $r(1,0)$ continues to approach its minimum but the depression propagates axially towards the two ends to accommodate the extra extension. When the depression finally reaches the two ends, the entire structure returns to a homogeneous cylinder of radius $1 / \sqrt{2.148}=0.682$ where 2.148 is the value of $\lambda$ corresponding to the Maxwell state $\left(S_{z z} \approx 6.417\right.$ is the middle dot in Fig. 10). We note that further softening occurs due to end effects, which is accompanied by further reduction of $S_{z z}$ from its Maxwell value. Afterwards the cylinder experiences uniform deformation and $S_{z z}$ increases with $\lambda$ (the right-most branch). The unloading path follows the dashed line in Fig. 10. It is noted that unloading does not entirely follow the loading curve, which is typical for phase-transformation problems. More precisely, when $\lambda$ is reduced to 1.710 (the right dot in Fig. 10), corresponding to the minimum in Fig. 5(a), a localized bulge initiates at $Z=L$, and $r(1, L)$ very quickly approaches its propagation value of 0.682 observed earlier. The propagation stage then follows as unloading continues. Eventually, the bulges reaches the two ends and the entire cylinder is of the same radius 1.174. The unloading curve joins the loading curve (the left-most branch) after a small jump. We remark that although the three dots representing the theoretical predictions do not exactly sit on the curves representing the simulation results, the relative errors are all less than $0.24 \%$. For instance, for the Maxwell value of $S_{z z}$ (the middle dot), the simulation and theoretical results are 6.405 and 6.417, respectively. This agreement is remarkable since only 10 mesh points are chosen in the radial direction in the numerical simulations. We also observe that the propagation value 6.417 of $S_{z z}$ is only marginally smaller than the initiation value 6.518. This is qualitatively consistent with the theoretical predictions based on the two boundaries in Fig. 7(b) between which localized solutions may exist.

For comparison we have also run a simulation with the same material and geometrical parameters except that the scaled length is reduced from $L=40$ to $L=2$. The corresponding result is represented by the dotted (black) line in Fig. 10. In this case the deformation remains homogeneous and the graph represents the numerical approximation of the exact relation (3.17). 


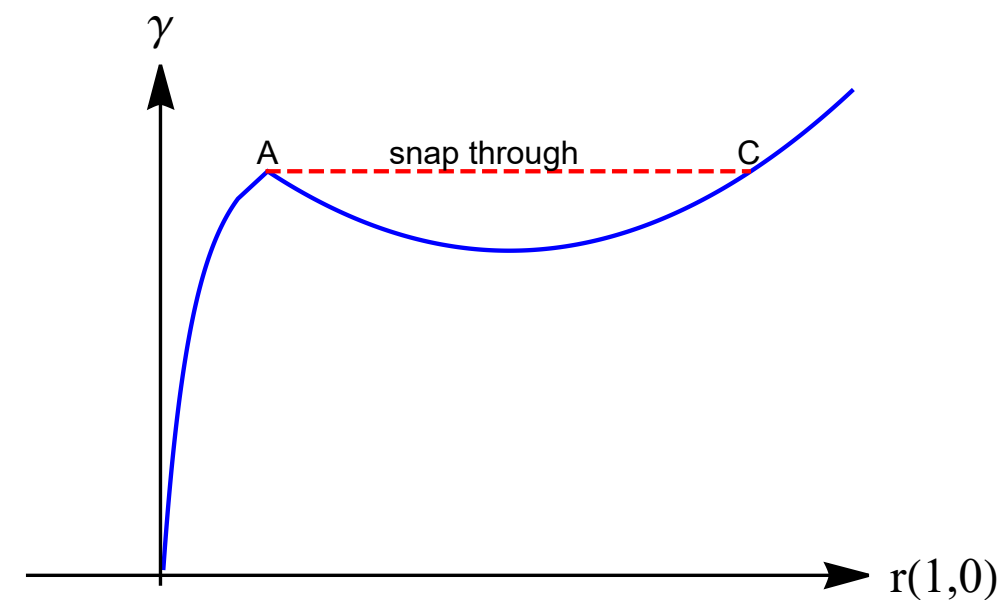

Figure 11: Likely dependence of $\gamma$ on $r(1,0)$ when ends of the cylinder are fixed.

Our theoretical predictions are at odds with the results of Henann \& Bertoldi (2014). In their Fig. 4(a) the instability appears to be a supercritical pitchfork bifurcation (see also Taffetani \& Ciarletta (2015)). This discrepancy may be explained as follows. Their results are obtained by fixing the length of the cylinder at its undeformed value and varying the surface tension monotonically without using the Riks method. Thus, their numerical scheme cannot deal with the type of softening behavior exhibited by the current problem. We believe that with a proper application of the Riks method, their diagram would be similar to the one shown in Fig. 11 (which is also consistent with Fig. 5(a) of Ye et al. (2019)). If reduction of $\gamma$ is not allowed in the simulation despite the formation of a localized depression/bulge, $r(1,0)$ would have to jump from the value at $A$ to the value at $C$, and as a result the stiffening behavior presented by Henann \& Bertoldi (2014) corresponds to the behavior on the right branch beyond point $C$. To verify this conjecture and also the predictions associated with Fig. 3(b) (i.e. the localized solution is a bulge when $\lambda>\lambda_{\min }$ and a depression when $\lambda>\lambda_{\min }$ ), we have repeated the calculations of Henann \& Bertoldi (2014) not only for the case of $\lambda=1$ but also for other values of $\lambda$. Fig. 12 shows the results for two typical cases: $\lambda=1$ and $\lambda=1.5$ for which localized necking and bulging take place, respectively. In each plot, the vertical part corresponds to the trivial solution. Since the cylinder is incompressible and the ends are fixed, the principal stretches and hence $r(1,0)$ do not change as $\gamma$ is increased. When $\gamma$ reaches the critical value predicted by (3.12), localized necking or bulging takes place depending on whether $\lambda$ is greater than or less than $\lambda_{\min }=1.23352$, exactly as our analysis predicts. In the case of necking (left figure), $r(1,0)$ decreases from its pre-bifurcation value, whereas in the case of bulging (right figure), $r(1,0)$ increases from its pre-bifurcation value. As remarked earlier, the jump in $r(1,0)$ associated with snap-through at the bifurcation point is clearly noticeable, manifested by a long horizontal segment starting from the bifurcation point.

In Fig. 13, we have shown the values of $\lambda$ not only at the center of the bulge or neck, but also at the ends of the cylinder after $\gamma$ is increased beyond its bifurcation value. Fig. 13(a) shows that for the special case when $\bar{\lambda}=\lambda_{\min }$, the bifurcation is genuinely supercritical and no snap-through takes place. This is consistent with our theoretical finding in Section 4.1.2 that no localized bulging or necking takes place when $\bar{\lambda}$ is fixed at this particular value. Fig. 13(b) compares the three cases corresponding to $\bar{\lambda}=1, \lambda_{\min }, 1.5$. It is seen that when $\bar{\lambda}=1$ or 1.5 , snap-through takes place and all the three cases adopt the same Maxwell state. However, the proportions of the two "phases" are different for different cases and are determined by the average stretch specified. 


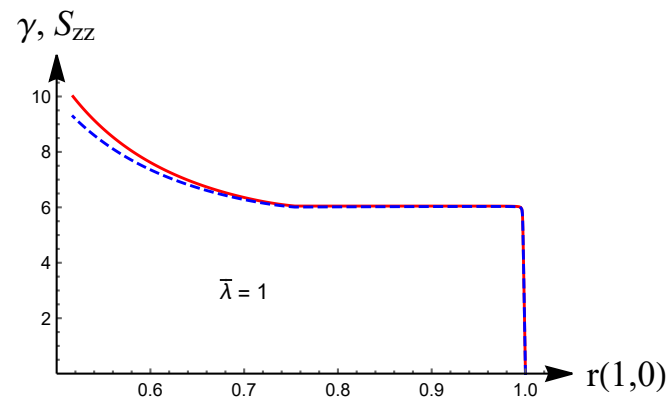

(a)

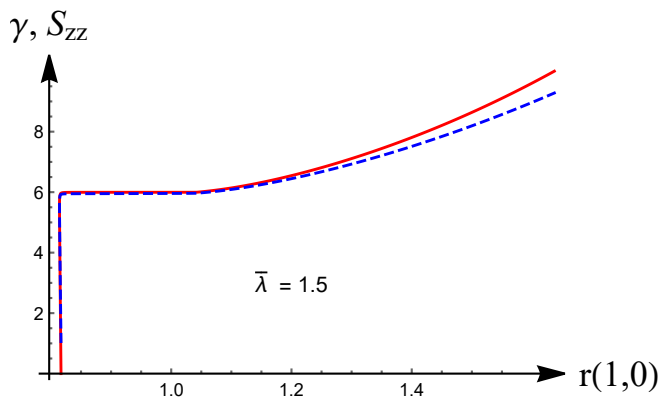

(b)

Figure 12: Numerical simulation results showing the dependence of $\gamma$ (solid line) and $S_{z z}$ (dashed line) on the radius at $Z=0$. (a) The cylinder is unstretched and ends are fixed prior to application of surface tension; (b) The cylinder is stretched by $50 \%$ and then ends are fixed prior to application of surface tension.

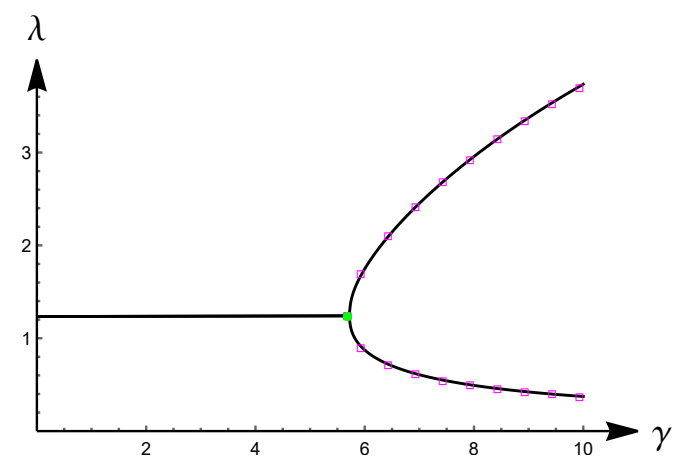

(a)

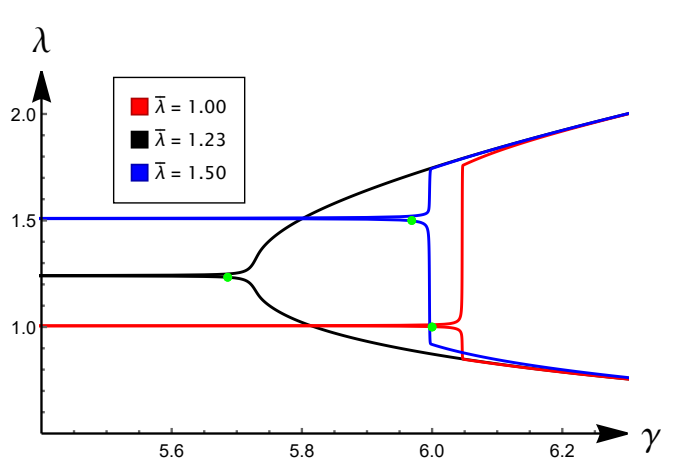

(b)

Figure 13: Dependence of the two Maxwell values of stretch on $\gamma$ when $\gamma$ is increased beyond its bifurcation value (marked by the green solid dot in each case). (a) $\bar{\lambda}=\lambda_{\min }=1.23$ in which case the uniform deformation bifurcates smoothly into a "two-phase" deformation without "snap through". Solid line: numerical simulation results; squares: analytical results based on (5.47). (b) Comparison of results for $\bar{\lambda}=1, \lambda_{\min }, 1.5$, showing the fact that when $\bar{\lambda}=1$ or 1.5 , the deformation would "snap through" to the same Maxwell state corresponding to $\bar{\lambda}=\lambda_{\min }$.

\section{Comparison to recent works}

Recent papers on the same topic have appeared after the initial submission of this paper, showing a great interest in the community for this problem. In Lestringant \& Audoly (2020), the authors derived a one-dimensional model for localized deformations of a solid cylinder with surface tension, generalizing the model derived in an earlier paper by Audoly \& Hutchinson (2016). This earlier model has recently been shown to be consistent with the amplitude equation, derived using the method of multiple scales, for the necking of a stretched cylinder without surface tension (Wang $\& \mathrm{Fu}, 2020$ ). We have checked and verified that the new model given by Lestringant \& Audoly (2020) can also be used to derive all the amplitude equations presented in the current paper with perfect agreement. Moreover, the preprint by Giudici \& Biggins (2020) overlaps with our analysis in Section 4.1.2 although a different reduction procedure was used. In particular, the equation (13) in Giudici \& Biggins (2020) is equivalent to our equation (4.29) after a substitution of the dependent variable and control parameter. Finally, we note that localized bulging/necking of cylindrical tubes under the combined action of axial stretching and surface tension has been examined by Wang (2020), with further insight provided by Emery \& Fu (2020). 


\section{Conclusion}

Localized bulging or necking is not usually possible in a hyperelastic solid cylinder under axial stretching but becomes possible when surface tension effect is taken into account. In this paper, we have explicitly determined the localized solutions that bifurcate from the primary uniform deformation. Three loading scenarios have been considered theoretically and verified numerically. When the averaged axial stretch, or equivalently the axial length, is fixed, localization will occur when surface tension reaches a critical value. This critical value is a function of the axial stretch and attains a minimum at $\lambda=\lambda_{\min }$ that depends on the material model used. We found that the localized solution corresponds to necking if $\lambda<\lambda_{\min }$ and bulging if $\lambda>\lambda_{\min }$, and that there further exists a thin layer around $\lambda=\lambda_{\text {min }}$ where localization takes place slightly earlier than predicted by the linear theory. At $\lambda=\lambda_{\min }$ exactly, localized solutions cease to exist and are replaced by "two-phase" deformations determined by Maxwell's equal area rule. Previous studies have all focussed on the case $\lambda=1$ and have presented bifurcation diagrams that look as if the bifurcation were supercritical. It is shown in this paper that such diagrams have missed a softening part and snap-through always occurs except when $\lambda=\lambda_{\min }$ for which the bifurcation is genuinely super-critical.

In the second scenario when the surface tension is fixed and axial force is allowed to vary, localized necking occurs when the axial force reaches a maximum. In the final scenario when the axial force is fixed (corresponding to a dead load at the ends) and the surface tension is increased gradually, the associated axial stretch decreases. When $\gamma$ reaches a maximum, bifurcation takes place and the bifurcated state corresponds to a bulge. The effect of surface tension and the possibility of bulging and necking is similar to the instability of an inflated elastic tube but different from the typical buckling/wrinkling instability found in solids where the new pattern emerges with a well-defined wavelength. This difference forces us to reach for more sophisticated asymptotic and numerical methods. The post-bifurcation analysis of these problems offers a rich class of complex behaviors that can be tested experimentally and we hope that our study will pave the way in that direction.

\section{References}

Abaqus (2013). ABAQUS Analysis Users Manual, version 6.13. Dassault Systmes, Providence, RI, USA.

Al-Izzi, S. C., Rowlands, G., Sens, P., \& Turner, M. S. (2018). Hydro-osmotic instabilities in active membrane tubes. Phy. Rev. Lett., 120, 138102.

Audoly, B., \& Hutchinson, J. W. (2016). Analysis of necking based on a one-dimensional model. J. Mech. Phys. Solids, 97, 68-91.

Bar-Ziv, R., \& Moses, E. (1994). Instability and "pearling"states produced in tubular membranes by competition of curvature and tension. Phy. Rev. Lett., 73, 1392-1399.

Bar-Ziv, R., Tlusty, T., , Moses, E., Safran, S. A., \& Bershadsky, A. (1999). Pearling in cells: A clue to understanding cell shape. Proc. Natl Acad. Sci., 96, 10140-10145.

Bar-Ziv, R., Tlusty, T., \& Moses, E. (1997). Critical dynamics in the pearling instability of membranes. Phy. Rev. Lett., 79, 1158-1161.

Barriere, B., Sekimoto, K., \& Leibler, L. (1996). Peristaltic instability of cylindrical gels. J. Chem. Phys., 105, 1735-1738. 
Bico, J., Reyssat, E., \& Roman, B. (2018). Elastocapillarity: When surface tension deforms elastic solids. Annu. Rev. Fluid Mech., 50, 629-659.

Boudaoud, A., \& Chaïeb (2003). Mechanical phase diagram of shrinking cylindrical gels. Phy. Rev. $E, 68,021801$.

Bush, J. W., \& Hu, D. L. (2006). Walking on water: biolocomotion at the interface. Annu. Rev. Fluid Mech., 38, 339-369.

Carroll, M. (2004). A representation theorem for volume-preserving transformations. Int. J. Nonlinear Mech., 39, 219-224.

Ciarletta, P. (2011). Generating functions for volume-preserving transformations. Int. J. Non-linear Mech., 46, 1275-1279.

Ciarletta, P., \& Ben Amar, M. (2012). Peristaltic patterns for swelling and shrinking of soft cylindrical gels. Soft Matter, 6, 1760-1763.

Datar, A., Ameeramja, J., Bhat, A., Srivastava, R., Mishra, A., Bernal, R., Prost, J., CallanJones, A., \& Pullarkat, P. A. (2019). The roles of microtubules and membrane tension in axonal beading, retraction, and atrophy. Biophy. J., 117, 880-891.

De Gennes, P. G., Brochard-Wyart, F., \& Quéré, D. (2004). Capillarity and wetting phenomena: drops, bubbles, pearls, waves. Springer Verlag.

Emery, D., \& Fu, Y.B. (2020). Localised bifurcation in soft cylindrical tubes under axial stretching and surface tension. Int. J. Solids Struct., under review.

Fong, H., Chun, I., \& Reneker, D. H. (1999). Beaded nanofibers formed during electrospinning. Polymer, 40, 4585-4592.

Fu, Y. B. (2001). Nonlinear stability analysis. In Nonlinear elasticity: theory and applications (eds $Y B$ Fu, $R W$ Ogden). Cambridge University Press, Cambridge.

Fu, Y. B., Liu, J. L., \& Francisco, G. S. (2016). Localized bulging in an inflated cylindrical tube of arbitrary thickness - the effect of bending stiffness. J. Mech. Phys. Solids, 90, 45-60.

Geng, Y. N., Huang, J. X., \& Fu, Y. B. (2016). Shape bifurcation of a pressurized ellipsoidal balloon. Int. J. Eng. Sci., 101, 115-125.

Giudici, A., \& Biggins, J.S. (2020). Ballooning, bulging and necking: an exact solution for longitudinal phase separation in elastic systems near a critical point. Phy. Rev. E, 102, 033007.

Goriely, A. (2017). The Mathematics and Mechanics of Biological Growth. Springer Verlag, New York.

Goriely, A., Geers, M. G. D., Holzapfel, G. A., Jayamohan, J., Jérusalem, A., Sivaloganathan, S., Squier, W., van Dommelen, J. A. W., Waters, S. L., \& Kuhl, E. (2015). Mechanics of the brain: perspectives, challenges, and opportunities. Biomech. Model. Mechanobiol., 14, 931-965.

Goriely, A., Vandiver, R., \& Destrade, M. (2008). Nonlinear Euler buckling. Proc. Roy. Soc. Lond. A, 464, 3003-3019.

Haragus, M., \& Iooss, G. (2011). Local bifurcations, center manifolds, and normal forms in infinitedimensional dynamical systems. Springer, London. 
Hemphill, M. A., Dauth, S., Yu, C. J., Dabiri, B. E., \& Parker, K. K. (2015). Traumatic brain injury and the neuronal microenvironment: a potential role for neuropathological mechanotransduction. Neuron, 85, 1177-1192.

Henann, D. L., \& Bertoldi, K. (2014). Modeling of elasto-capillary phenomena. Soft Matter, 10, 709-717.

Iooss, G., \& Adelmeyer, M. (1999). Topics in Bifurcation Theory and Applications. World Scientific, Singapore.

Kilinc, D., Gallo, G., \& Barbee, K. A. (2008). Mechanically-induced membrane poration causes axonal beading and localized cytoskeletal damage. Exp. Neur., 212, 422-430.

Kilinc, D., Gallo, G., \& Barbee, K. A. (2009). Interactive image analysis programs for quantifying injury-induced axonal beading and microtubule disruption. Comp. Meth. Progr. Biom., 95, $62-71$.

Kirchgässner, K. (1982). Wave solutions of reversible systems and applications. J. Diff. Eqns., 45, 113-127.

Lang, G. E., Waters, S. L., Vella, D., \& Goriely, A. (2017). Axonal buckling following stretch injury. J. Elast., 129, 239-256.

Lestringant, C., \& Audoly, C. (2020). A one-dimensional model for elasto-capillary necking. Proc. Roy. Soc. Lond. A, https://doi.org/10.1098/rspa.2020.0337.

Levich, V., \& Krylov, V. (1969). Surface-tension-driven phenomena. Ann. Rev. Fluid Mech., 1, 293-316.

Markin, V. S., Tanelian, D. L., Jersild Jr., R. A., \& Ochs, S. (1999). Biomechanics of stretch-induced beading. Biophy. J., 76, 2852-2860.

Matsuo, E. S., \& Tanaka, T. (1992). Patterns in shrinking gels. Nature, 358, 482-485.

Mora, S., Phou, T., Fromental, J.-M., Pismen, L. M., \& Pomeau, Y. (2010). Capillarity driven instability of a soft solid. Phys. Rev. Lett, 105, 214301.

Mozaffari, K., Yang, S. Y., \& Sharma, P. (2019). Surface Energy and Nanoscale Mechanics, in Handbook of Materials Modeling (eds W. Andreoni and S. Yip). Springer, Cham.

Naraghi, M., Chasiotis, L., Kahn, H., Wen, Y., \& Dzenis, Y. (2007). Mechanical deformation and failure of electrospun polyacrylonitrile nanofibers as a function of strain rate. Appl. Phys. Lett., 91, 151901.

Nelson, P., \& Powers, T. (1995). Dynamical theory of the pearling instability in cylindrical vesicles. Phy. Rev. Lett., 74, 3384-3387.

Ochs, S., Pourmand, R., Jersild Jr, R. A., \& Friedman, R. N. (1997). The origin and nature of beading: a reversible transformation of the shape of nerve fibers. Progr. Neurobiol., 52, 391-426.

Plateau, J. (1873). Statique expérimentale et théorique des liquides soumis aux seules forces moléculaires volume 2. Gauthier-Villars.

Pullarkat, P. A., Dommersnes, P., Fernández, P., Joanny, J.-F., \& Ott, A. (2006). Osmotically driven shape transformations in axons. Phy. Rev. Lett., 96, 048104. 
Rayleigh, L. (1892). On the instability of a cylinder of viscous liquid under capillary force. Phil. Mag. Series 5, 34, 145-154.

Sattler, R., Wagneer, C., \& Eggers, J. (2008). Blistering pattern and formation of nanofibers in capillary thinning of polymer solutions. Phy. Rev. Lett., 100, 164502.

Style, R. W., Jagota, A., Hui, C.-Y., \& Dufresne, E. R. (2017). Elastocapillarity: Surface tension and the mechanics of soft solids. Ann. Rev. Condens. Matter Phys., 8, 99-118.

Taffetani, M., \& Ciarletta, P. (2015). Beading instability in soft cylindrical gels with capillary energy: weakly non-linear analysis and numerical simulations. J. Mech. Phy. Solids, 81, 91-120.

Wang, L. (2020). Axisymmetric instability of soft elastic tubes under axial load and surface tension. Int. J. Solids Struct., 191-192, 341-350.

Wang, M., \& Fu, Y. B. (2020). Necking of a hyperelastic solid cylinder under axial stretching: evaluation of the infinite-length approximation. Int. J. Eng. Sci., submitted.

Wang, S. B., Guo, G. M., Zhou, L., Li, L. A., \& Fu, Y. B. (2019). An experimental study of localized bulging in inflated cylindrical tubes guided by newly emerged analytical results. $J$. Mech. Phy. Solids, 124, 536-554.

Wang, T., Xu, F., Huo, Y., \& Potier-Ferry, M. (2018). Snap-through instabilities of pressurized balloons: Pear-shaped bifurcation and localized bulging. Int. J. Nonlinear Mech., 98, 137-144.

Wilkes, E. (1955). On the stability of a circular tube under end thrust. Q. J. Mech. Appl. Math., 8, 88-100.

Wineman, A. (2005). Some results for generalized neo-hookean elastic materials. Int. J. Non-Linear Mech., 40, 271-279.

Wolfram Research Inc (2019). Mathematica: version 12. Wolfram Research Inc, Champaign, IL.

Xuan, C., \& Biggins, J. (2016). Finite-wavelength surface-tension-driven instabilities in soft solids, including instability in a cylindrical channel through an elastic solid. Phy. Rev. E, 94, 023107.

Xuan, C., \& Biggins, J. (2017). Plateau-Rayleigh instability in solids is a simple phase separation. Phy. Rev. E, 95, 053106.

Ye, Y., Liu, Y., \& Fu, Y. B. (2019). Weakly nonlinear analysis of localized bulging of an inflated hyperelastic tube of arbitrary wall thickness. J. Mech. Phy. Solids, 135, 103804. 